\title{
Review Article Physics of Internal Photoemission and Its Infrared Applications in the Low-Energy Limit
}

\author{
Y. F. Lao ${ }^{1}$ and A. G. U. Perera ${ }^{1,2}$ \\ ${ }^{1}$ Department of Physics and Astronomy, Georgia State University, Atlanta, GA 30303, USA \\ ${ }^{2}$ Center for Nano-Optics (CeNO), Georgia State University, Atlanta, GA 30303, USA \\ Correspondence should be addressed to A. G. U. Perera; uperera@gsu.edu
}

Received 13 September 2015; Accepted 29 December 2015

Academic Editor: Jung Y. Huang

Copyright ( 2016 Y. F. Lao and A. G. U. Perera. This is an open access article distributed under the Creative Commons Attribution License, which permits unrestricted use, distribution, and reproduction in any medium, provided the original work is properly cited.

\begin{abstract}
Internal photoemission (IP) correlates with processes in which carriers are photoexcited and transferred from one material to another. This characteristic allows characterizing the properties of the heterostructure, for example, the band parameters of a material and the interface between two materials. IP also involves the generation and collection of photocarriers, which leads to applications in the photodetectors. This review discusses the generic IP processes based on heterojunction structures, characterizing p-type band structure and the band offset at the heterointerface, and infrared photodetection including a novel concept of photoresponse extension based on an energy transfer mechanism between hot and cold carriers.
\end{abstract}

\section{Introduction}

Internal photoemission (IP) spectroscopy is an attractive method [1-4] for studying the properties of materials and optical processes that take place at the interface of two materials. IP refers to such a case where carriers are photoexcited and transferred from one material to another by passing through an interface. Photoexcitation occurs in the absorber (referred to as IP emitter) before photoemission. The photoexcited holes with high energies originate from those at the states around the Fermi level. By having higher energy than the photoemission threshold, these carriers can come across the penitential barrier; in other words, photocarriers must be transferred from the energy band of the emitter to that of the barrier material. This process typically involves scatterings through which the excited holes are directed to pass over the emitter-barrier interface. Optical excitation includes direct and indirect transitions. The type of transitions that contributes to quantum yield (defined as number of carriers being collected per incident photon) relies on the energy of the incident photon. In the vicinity of the photoemission threshold, carriers must stay at states with energies nearly at the same level of the potential barrier before emission. This results in a higher probability of the occurrence of indirect transitions. However, under the excitation of photons with energies much greater than the threshold energy, direct transitions dominate over the indirect transitions in contributing the quantum yield significantly. Although many of IP studies were reported before [1], IP is still attractive, in particular, with recent demonstration of its advantages in characterizing new material systems $[3,4]$. Based on the above-mentioned principle, the use of the IP effect lies in at least the following areas: (1) studying the band parameters of materials, such as the band structure; (2) studying the band offset at the interface of a heterostructure; (3) development of IP-based photodetectors.

The internal photoemission is characterized by the quantum yield [2], which can be obtained as being the multiplication of $R$ and $h \nu$, where $R$ and $h \nu$ are the responsivity and the energy of the photon, respectively. Photoresponse in the energy regime near the IP threshold comes with two features determined by the optical transitions in the active region which create photocarriers and the escape of photocarriers which leads to photocurrents. The escape capability can be mitigated by the loss in the energy or redirection of the carrier as a result of scattering with phonon. The onset of the carrier-phonon scattering depends on the excess kinetic energy of photocarriers [1]. It is expected 


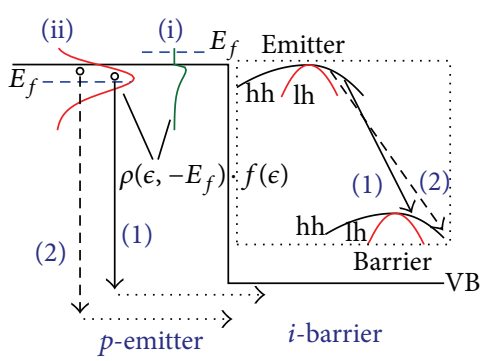

(a)

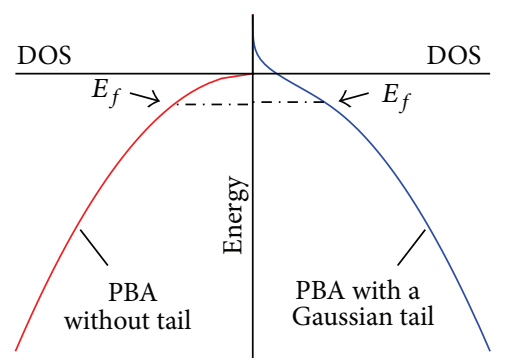

(b)

FIGURE 1: (a) Schematic band diagram of a $p$-emitter/i-barrier structure. $\rho\left(\epsilon,-E_{f}\right) f(\epsilon)$ is the multiplication of the energy distribution $(\rho)$ and the FD function $(f)$. (i) and (ii) correspond to the nondegenerate and degenerate doping, respectively. IP processes labeled as (1) and (2) consist of optical transitions in the emitter and the escaping of holes from the emitter to the barrier. (b) DOS with and without the band tailing effect.

that this occurs at the above-threshold regime, and after excitation, the excess kinetic energy of carriers should be greater than the phonon energy. Upon scatterings, a portion of photocarriers could remain in the absorber, unable to escape, which leads to degradation in the photoresponse. A detailed study on this should benefit future improvement of the device performance. The IP approach studying the scattering effects has been recently demonstrated [8] through fitting of the near-threshold quantum yield spectra.

In an IP experiment, photoemitted carriers are collected and detected as a photocurrent varying with the photon energy. The IP effect for photodetection requires the use of a heterostructure. An important concept recently demonstrated [9] is the photoresponse extension triggered by introducing a hot-cold carrier energy mechanism. The photoresponse is substantially extended without the need of reducing the IP threshold energy.

In this review, we discuss the IP spectroscopy as a method to characterize semiconductors and heterostructures and use the IP effect to develop photodetectors.

\section{IP Characterization of Band Parameters}

2.1. The Theory of the IP Spectroscopy. The theoretical basis of IP was initially reported in Fowler's works [13] on external photoemission of electrons (from metal to vacuum). The original form of the quantum yield $Y$ can be expressed as

$$
Y \sim f(\mu) \cdot(k T)^{2}
$$

which contains a $T$-dependent term. $f(\mu)$ has the following expression [13]:

$$
\begin{aligned}
& f(\mu) \\
& = \begin{cases}e^{\mu}-\frac{e^{2 \mu}}{4}+\frac{e^{3 \mu}}{9}-\cdots & (\mu \leqslant 0) \\
\frac{\pi^{2}}{6}+\frac{\mu^{2}}{2}-\left(e^{-\mu}-\frac{e^{-2 \mu}}{4}+\frac{e^{-3 \mu}}{9}-\cdots\right) & (\mu \geqslant 0),\end{cases}
\end{aligned}
$$

where $\mu \equiv(h v-\Delta) / k T$ and $h v$ is the photon energy. Fowler's yield function can be actually reduced [14] to be independent of $T$ when $h v>\Delta+3 k T$; that is,

$$
Y(h \nu) \sim(h \nu-\Delta)^{2} .
$$

Fowler's yield function is mainly used to fit the IP thresholds. Despite the fact that Fowler's yield function was originally derived for external photoemission, it has been applied [15] to many IP cases. By taking into account the fact that semiconductors have a different band structure compared to metal, exponent " 2 " in (3) is replaced with a parameter $p[16-$ 19]. The value of $p$ was reported varying between 1 and 3 [19]. By taking $p$ as a fitting parameter, the physical meaning of the yield function becomes unclear. Furthermore, the effect of the temperature on the IP process is ignored, which could cause underestimate of $\Delta$. This result is significant when the yield function applied to the low-energy limit, for example, in the energy range of $<1 \mathrm{eV}$.

To obtain accurate IP thresholds, temperature-dependent IP spectroscopy (TDIPS) has been developed. Figure 1(a) shows the TDIPS principle for a $p$-type structure, which includes two doping concentrations, under which $E_{f}$ can be found to be (i) above and (ii) below the top of the valence band (VB). By absorbing photons, photoexcited holes with high energies from the emitter escape over the potential barrier. The photoemission process of carrier transport from the emitter to the barrier is shown in the inset of Figure 1(a). During this process, there exist photon absorption and scatterings. The scattering is crucial for the photoexcited holes to be transferred across the emitter-barrier interface. Both direct and indirect transitions can lead to photoexcitation and have the primary contributions to the IP yield in the energy regime near and greater than the IP threshold, respectively.

To obtain the band offsets, the line shape of the spectral quantum yield should be considered. It should be noted that the IP yield concerns with the primary processes including the carriers' energies and the escape probabilities of these carriers, which can be evaluated by considering the photoexcitation of holes via intervalence band (IVB) transitions [12] $\left(\rho\left(\epsilon, h \nu-E_{f}\right)\right)$ and the escape of the holes $(P(\epsilon, \Delta))[20]$. These considerations lead to the following expression:

$$
\begin{aligned}
Y(h v)= & Y_{0}(k T) \\
& +C_{0} \int_{\Delta}^{\infty} f(\epsilon, h v) \rho\left(\epsilon, h v-E_{f}\right) P(\epsilon, \Delta) d \epsilon,
\end{aligned}
$$

where $\rho\left(\epsilon, h \nu-E_{f}\right)$ is an energy distribution function and $P(\epsilon, \Delta)$ a probability function. $C_{0}$ is a constant. $\epsilon$ is the energy 
of the hole. $\Delta$ is the barrier height corresponding to an energy difference between the Fermi level and the barrier. Equation (4) is deduced in terms of the degenerate (highly) doped emitters; by changing the sign of $E_{f}$, it can be used for nondegenerate doping. The Fermi-Dirac (FD) statistics tell that carriers are at the energy states above the Fermi level at finite temperatures. To this end, $f(\epsilon, h \nu)=\left[1+e^{(\epsilon-h \nu) / k T}\right]^{-1}$ is used to describe the the distribution of carriers, provided that the energy states of photoexcited holes are the same as those before photoexcitation.

Equation (4) contains a term $Y_{0}$ which is the background signal, for example, the thermionic background. It can be determined by giving $Y_{0}$ the value of the experimental yield in the energy range less than the value of $\Delta$.

The function $P(\epsilon, \Delta)$ is calculated by an escape-cone model $[20,21]$. For a successful escaping over a barrier, the normal (to the interface) momentum of carriers must be greater than the potential barrier. Correspondingly, the energy states on a spherical Fermi cap in the $\mathbf{k}$ space are accounted to calculate $P(\epsilon, \Delta)$, which gives a value proportional to $(\epsilon-\Delta)$ for $\epsilon \geq \Delta$ and 0 for $\epsilon<\Delta$.

The energy distribution $\left[\rho\left(\epsilon, \epsilon_{0}\right)\right]$ of photocarriers has a primary influence on the quantum yield. By considering the fact that the direct transitions are associated with the joint density of states (JDOS) and indirect transitions are mainly determined by the occupation of initial states [22], the density of states (DOS) of the VB can be used for $\rho\left(\epsilon, \epsilon_{0}\right)$. For the transitions taking place near the $\Gamma$ point, the use of the parabolic-band approximation (PBA) leads to $\rho\left(\epsilon, \epsilon_{0}\right) \sim$ $\left(\epsilon-\epsilon_{0}\right)^{1 / 2}$.

As the emitters are doped, the IP model should also take into account the doping-inducted effects, for example, the band tailing states. Potential fluctuations can be induced by dopants, leading to band tailing states at the top of the VB. As shown in Figure 1(b), the Kane model [23] is used to modify the PBA DOS. Dopants-induced changes in DOS have a primary influence on the quantum yield near the energy of the IP threshold; the quantum yield is increased for $h v>$ $E_{f}+\Delta$ as a consequence of contribution from the states in the forbidden gap and is slightly reduced for $h v<E_{f}+\Delta$. The consequence of this is the smaller $\Delta$ determined by fitting to the yield spectra.

The process to determine the IP threshold is primarily influenced by the IP probability $P(\epsilon, \Delta)$. In addition, $\rho\left(\epsilon, \epsilon_{0}\right)$ is associated with the optical transitions. By taking into account the slowly varying nature of $f$ and $P$, and thus the primary effect of $\rho$ on the yield, the derivative can be obtained as $d Y / d(h \nu) \sim \rho\left(\Delta-h \nu, E_{f}\right)$. Therefore, the second derivative

$$
\frac{d^{2} Y}{d(h \nu)^{2}} \sim \frac{d(\text { JDOS })}{d(h \nu)},
$$

where $\rho$ can be obtained using JDOS when direct IVB transitions are considered. This indicates a relationship between the band structure and the IP yield.

2.2. IP Determination of the Spin-Orbit Splitting and the Participation of Phonons in Indirect Intervalence Band Transitions. As shown in Figure 2(a), indirect transitions take place via an intermediate state, which can be in the same VB or in a nearby band. The phonon-assisted indirect interband absorption can be easily measured [24, 25]. However, phonon participating in indirect IVB transitions is difficult to be measured, because it is usually featureless [26, 27] (e.g., Figure 2(b)). IP takes place when the photoexcited carriers (in the photon absorber (emitter)) have the kinetic energies greater than the barrier [2]. To escape, these photoexcited carriers must transmit from the absorber to the barrier. Because of this, indirect transitions can lead to remarkable features due to their higher probabilities of occurrence than the direct transitions [22].

The quantum yield spectra of different p-type GaAs/ $\mathrm{AlGaAs}$ samples with different $\mathrm{Al}$ fractions are shown in Figure 2(c) which shows the quantum yield spectra. It can be seen that these samples have similar spectral profiles between 0.3 and $0.45 \mathrm{eV}$. The fluctuations at energies greater than $0.45 \mathrm{eV}$ are due to optical interference. It is noted that the features between 0.3 and $0.45 \mathrm{eV}$ are independent of the biases, the height, and the shape of the potential barrier. These features can be considered to be related to the $\mathrm{SO}-\mathrm{HH}$ hole transitions.

The differentiated quantum yield spectra are shown in Figures 3(a)-3(c). Different features observed have the onset in consistence with the double-differential peaks. The peak at $\sim 0.34 \mathrm{eV}$ on the second derivative spectra has the correlation with the SO-HH singularity. The verification of this can be done by calculating $1 / \nabla_{k} \sum_{i, j}\left[E_{i}(\mathbf{k})-E_{j}(\mathbf{k})\right]$, as shown in Figure 3(d). The weak feature of the SO-HH singularity agrees with the experimental data.

The value of $\Delta_{0}$ can be determined $[28,29]$ by resolving the critical points (interband transitions): $E_{0}$ and $E_{0}+\Delta_{0}$, which correspond to the $\mathrm{HH}$ to $\mathrm{CB}$ transition and the $\mathrm{SO}$ to $\mathrm{CB}$ transition, respectively. In contrast to this, a direct determination of $\Delta_{0}$ is advantageous to the studies of its variation with the doping concentration (Figure 3) and temperature (Figure 4(a)). Figure 4(b) shows the determined values that nearly remain the same, with an average of $0.3392 \pm 0.0003 \mathrm{eV}$. The result agrees with reported values in the past, that is, $0.390-0.341 \mathrm{eV}$ [30-32]. It is difficult to determine the doping dependency of the $\Delta_{0}$ by using the interband transitions [27], because DOS at the $\Gamma$ point can be significantly modified by the band tailing states. However, the value of $\Delta_{0}$ can be obtained by the two-thirds rule [i.e., $\Delta_{1}=(2 / 3) \Delta_{0}$ ] [33], by which, a constant $\Delta_{0}$ can be found from the synchronous variation of the interband critical points $E_{1}\left(\mathrm{HH}\right.$ to $\mathrm{CB}$ ) and $E_{1}+\Delta_{1}(\mathrm{LH}$ to $\mathrm{CB})$ (at larger $\mathbf{k}$ ) with doping concentrations [34].

The energies of various phonons can be determined by the internal photoemission spectroscopy. Figure 3(a) shows the two strong features at $\sim 0.37 \mathrm{eV}$ and $0.44 \mathrm{eV}$. By considering that the features correspond to the energies of $\Delta_{0}+E_{\mathrm{ph}}$ (where $E_{\mathrm{ph}}$ is the phonon energy), these two features correlate with $\operatorname{LO}(\Gamma)$ and $3 \times \mathrm{TO}(\Gamma)$. Following a similar procedure, a variety of phonons can be determined as shown in Table 1 . It should be noted that the most important phonons, $\mathrm{LO}(\Gamma)$ and $3 \times \mathrm{TO}(\Gamma)$, have the significant contributions to the quantum yield. The result was also observed in previous studies on the hole-optical phonon interactions [35-37]. 


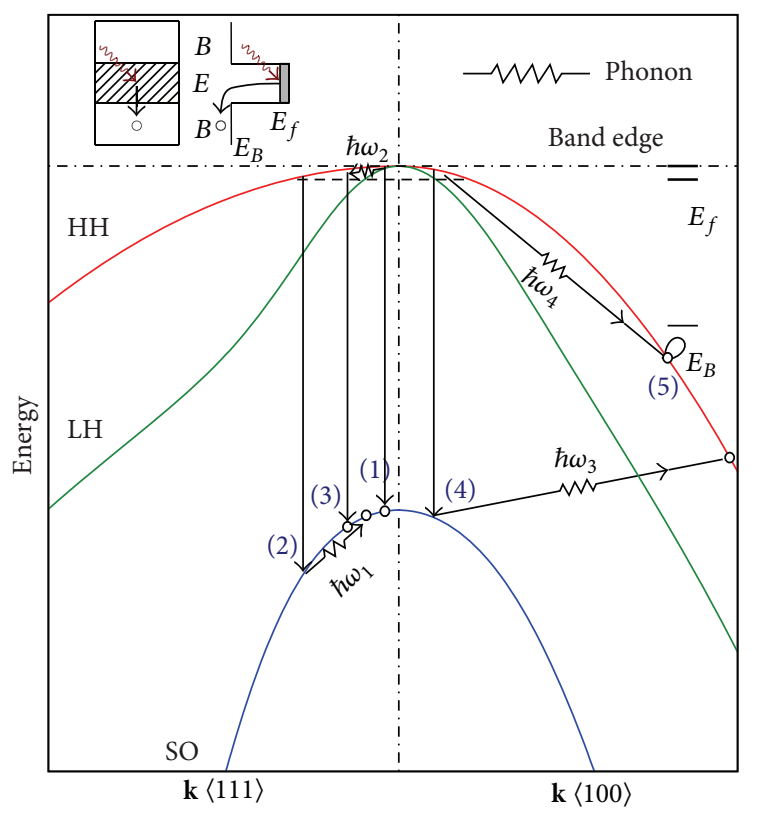

(a)

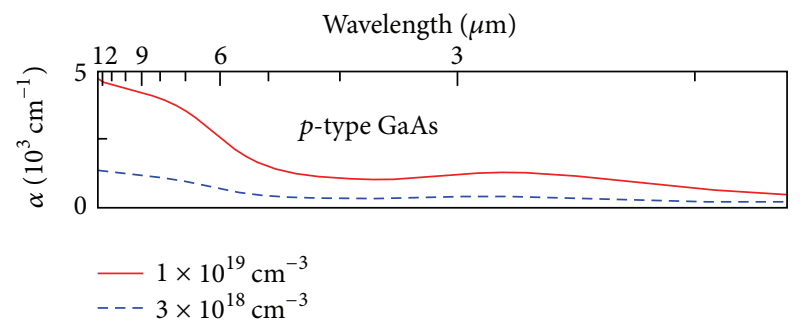

(b)

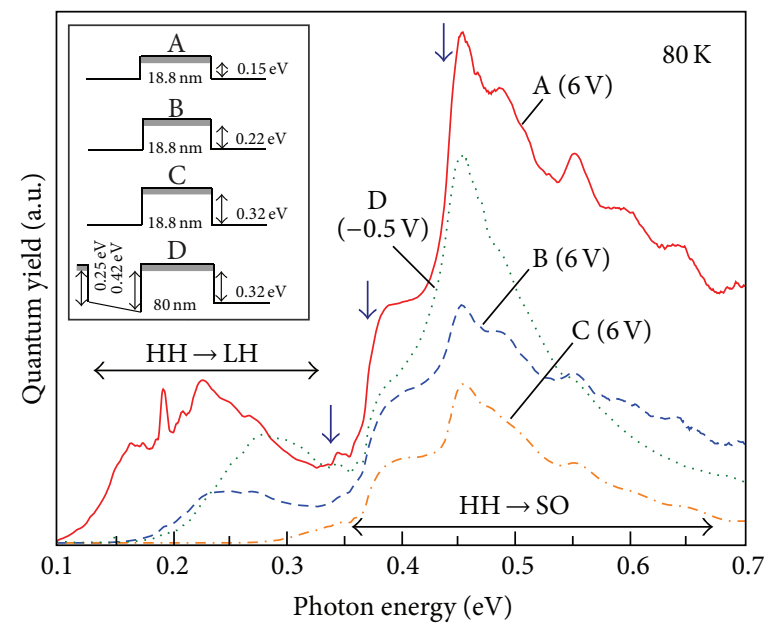

(c)

FiguRE 2: (a) Schematic of different intervalence band optical transitions. (1)-(5) correspond to (1) direct transition; (2) direct transition + phonon emission $\left(\hbar \omega_{1}\right)$; (3) phonon absorption $\left(\hbar \omega_{2}\right)+$ direct transition; (4) direct transition + phonon emission $\left(\hbar \omega_{3}\right)$ (interband scattering); and (5) phonon absorption $\left(\hbar \omega_{4}\right)+$ intraband photon absorption. Inset plots the optical processes taking place in an emitter $(E) /$ barrier $(B)$ heterojunction. (b) Optical absorption of $p$-type GaAs. (c) Quantum yield spectra of different $p$-type $\mathrm{GaAs} / \mathrm{Al}_{x} \mathrm{Ga}_{1-x} \mathrm{As}_{\text {seterojunctions. }}$ Inset shows the flat-band (VB) diagrams [5] at zero bias. The doping concentrations are $3 \times 10^{18} \mathrm{~cm}^{-3}(\mathrm{~A}, \mathrm{~B}$, and $\mathrm{C})$ and $1 \times 10^{19} \mathrm{~cm}^{-3}(\mathrm{D})$. The vertical arrows indicate the features that remain the same in different samples.

2.3. IP Studies of the Band Offsets of Type-II InAs/GaSb Superlattice Structure. As shown in the $p \mathrm{~B} p$ T2SL detector (Figure 5(a)) where two $p$-type InAs/GaSb T2SL absorbers responsible for $\mathrm{mid} /$ long-wave infrared (MWIR/LWIR) absorption are separated by a B-region consisting of an InAs/AlSb T2SL, different optical transitions can be resolved by switching the bias polarity, as shown in Figure 5(b). Possible transitions include those occurring across the forbidden gaps of the superlattice absorbers (I), the B-region
(II), and the VB offset between the VB of the absorber and the B-region interface (III), the latter two of which can be observed in the higher photon energy regime. This is shown in the quantum yield $(Y)$ spectra plotted in Figure 5(c).

Fittings to quantum yield spectra in different nearthreshold energy regimes were carried out, as shown in Figure 5(c). A threshold energy corresponding to one specific transition is determined by the fact that the band offset has the value between the band gaps of absorbers and B-region. 


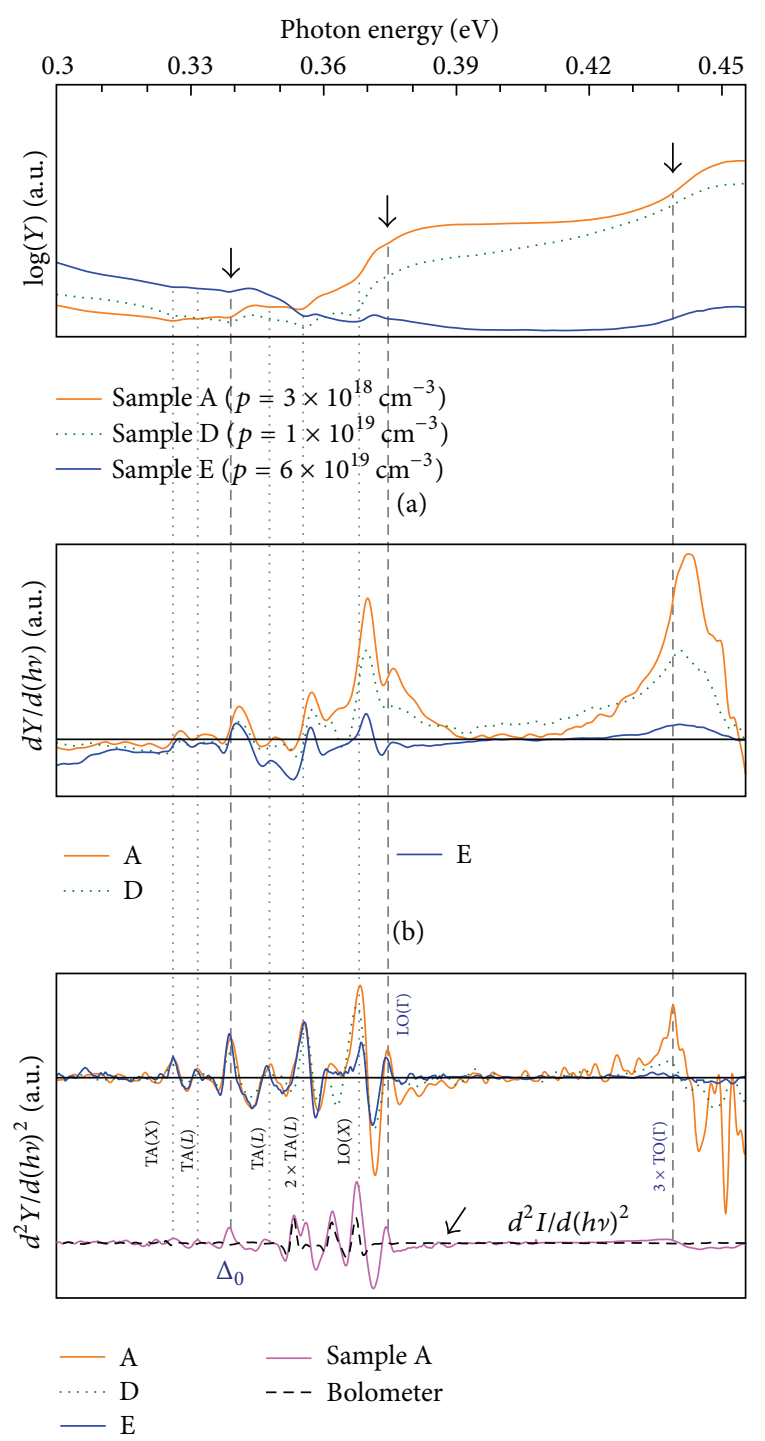

(c)

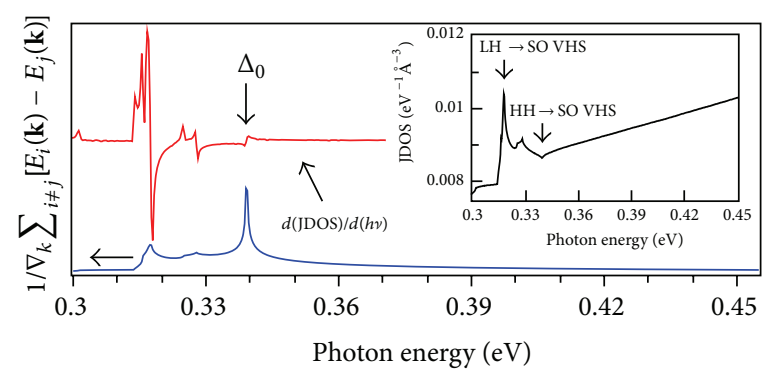

(d)

Figure 3: (a) Quantum yield ( $Y$ ); (b) first and (c) second derivatives of quantum yield spectra for samples A (6 V, $80 \mathrm{~K}), \mathrm{D}(-0.5 \mathrm{~V}, 80 \mathrm{~K})$, and $\mathrm{E}(0.05 \mathrm{~V}, 50)$. The structure of sample $\mathrm{E}$ is the same as A, except for its emitter doping concentration $\left(6 \times 10^{19} \mathrm{~cm}^{-3}\right)$. The vertical lines correspond to features listed in Table 1. Also shown in (c) (at the bottom) is bolometer (background) spectra. (d) Calculated value of $1 / \nabla_{k} \sum_{i \neq j}\left[E_{i}(\mathbf{k})-E_{j}(\mathbf{k})\right]$ based on a $\mathbf{k} \cdot \mathbf{p}$ model [6]. The Van Hove singularities (VHS) of intervalence band transitions are indicated.

This determines the band gaps (average) of the LWIR and MWIR absorbers to be $0.117 \pm 0.002 \mathrm{eV}$ and $0.157 \pm 0.001 \mathrm{eV}$, respectively, agreeing with the nominal values of $0.103 \mathrm{eV}$ and $0.159 \mathrm{eV}$, respectively [41]. Based on the obtained VB offset
$[0.661( \pm 0.002) \mathrm{eV}]$, the CB offset between the LWIR absorber and the B-region is determined to be $0.004 \pm 0.004 \mathrm{eV}$, close to the designed value $(0.009 \mathrm{eV})$ [41]. Such a small value should barely affect the transport of minority electrons. 


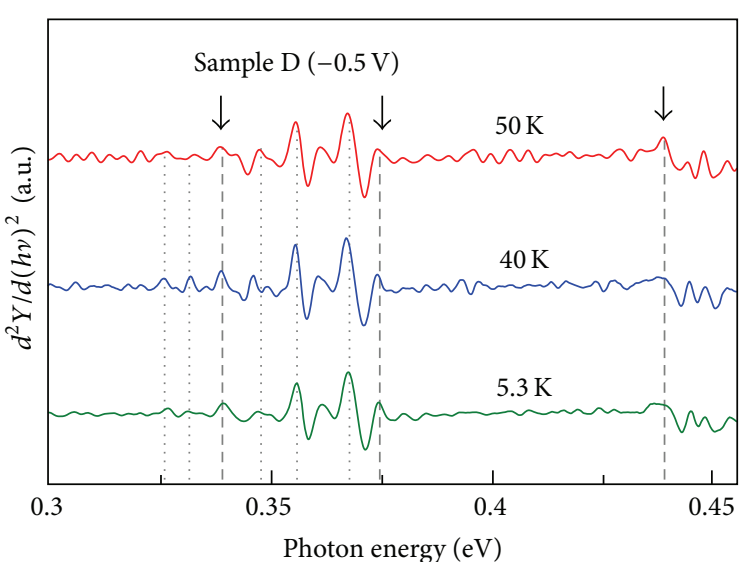

(a)



(b)

Figure 4: (a) Second yield derivative of the quantum yield spectra at different temperatures. (b) Temperature and doping dependence of $\Delta_{0}$ for GaAs. Data of $\Delta_{0}$ adopted from Table 3 of [7] are shown for comparison.

TABLE 1: Phonons of GaAs ( $80 \mathrm{~K})$ determined by internal photoemission spectroscopy. $\Delta_{0}$ is $339.2 \pm 0.3 \mathrm{meV}$. The numbers in parenthesis are uncertainties.

\begin{tabular}{|c|c|c|c|c|c|}
\hline Features $(\mathrm{meV})$ & $\Delta_{0}-13.0$ & $\Delta_{0}-7.4$ & $\Delta_{0}$ & $\Delta_{0}+8.7$ & $\Delta_{0}+16.3$ \\
\hline Phonons & $\mathrm{TA}(X)$ & $\mathrm{TA}(L)$ & - & $\mathrm{TA}(L)$ & $2 \times \mathrm{TA}(L)$ \\
\hline Features $(\mathrm{meV})$ & $\Delta_{0}+28.9$ & $\Delta_{0}+35.4$ & $\Delta_{0}+99.4$ & & \\
\hline \multirow[t]{2}{*}{ Phonons } & $\mathrm{LO}(X)$ & $\mathrm{LO}(\Gamma)$ & $3 \times \mathrm{TO}(\Gamma)$ & & \\
\hline & $\mathrm{TO}(\Gamma)$ & $\mathrm{LO}(\Gamma)$ & $\mathrm{LO}(X)$ & $\mathrm{TA}(X)$ & $\mathrm{TA}(L)$ \\
\hline This work (meV) & $33.1(0.2)$ & $35.4(0.2)$ & $28.9(0.2)$ & $12.2(0.8)$ & $8.1(0.5)$ \\
\hline Waugh and Dolling ${ }^{a}$ & $33.2(0.3)$ & $35.4(0.8)$ & $29.9(0.6)$ & $9.75(0.06)$ & $7.70(0.06)$ \\
\hline Blakemore $^{\mathrm{b}}$ & 33 & 35.5 & 30 & 8 & - \\
\hline Giannozzi et al..$^{c}$ & 33.6 & 36.1 & 29.8 & 10.2 & 7.8 \\
\hline Steiger et al. $^{\mathrm{d}}$ & 33.2 & 36.1 & 28.9 & 8.9 & 8.8 \\
\hline
\end{tabular}

a $[38]$.

${ }^{b}$ Extracted from multiple phonon features; see Table XI of [32].

${ }^{c}[39]$.

$\mathrm{d}_{[40] \text {. }}$

It should be noted that photoemission probabilities should vary, in order to fit the band gap or band offset (see Figure 5(c)). With respect to IP at an interface at which photocarriers need to overcome a potential barrier, $P(\epsilon, \Delta)$ equals $(\epsilon-\Delta)$ for $\epsilon \geq \Delta$ and 0 for $\epsilon<\Delta$ [2], according to an escape-cone model [20]. It is apparent, however, that photocarriers excited in the MWIR/LWIR T2SL absorber can freely move towards the direction of photocurrents until being collected. This means $100 \%$ transmission probability. The IP fittings using different $P(\epsilon, \Delta)$ plotted in Figure 5(c) agree with the above operating mechanism. The inset of Figure 5(c) shows the fitting based on $P(\epsilon, \Delta)=(\epsilon-\Delta)$ that leads to an incorrect spectral profile compared to $P(\epsilon, \Delta)=1$, thus not being able to explain the experimental data. The use of varied $P(\epsilon, \Delta)$ in consistence with optical processes under operation is a justification of the applicability of IP spectroscopy to T2SL structures.
2.4. Study of HgCdTe Infrared Photodetectors. A schematic of the $\mathrm{Hg}_{1-x} \mathrm{Cd}_{x} \mathrm{Te} / \mathrm{Hg}_{1-y} \mathrm{Cd}_{y} \mathrm{Te}$ sample is shown in Figure 6(a), consisting of a thin $(0.32 \mu \mathrm{m})$ top $\mathrm{Hg}_{0.68} \mathrm{Cd}_{0.32} \mathrm{Te}$ layer and a thick $(7.33 \mu \mathrm{m}) \mathrm{Hg}_{0.78} \mathrm{Cd}_{0.22} \mathrm{Te}$ absorber, grown on the Si substrate. Two ohmic contacts are made to the individual $\mathrm{HgCdTe}$ layers. Backside illumination was used for all of the measurements. Figure 6(b) shows the typical quantum yield spectra at 5.3 and $78 \mathrm{~K}$. The undulation is related to the optical interference inside the substrate. The low-energy cut-off is due to the escape of photocarriers from the absorber (originating from the optical transition schematically shown in the inset). Features associated with the band gap of the $\mathrm{Hg}_{0.68} \mathrm{Cd}_{0.32}$ Te layer, which should be around 0.28 and $0.29 \mathrm{eV}$ for 5.3 and $78 \mathrm{~K}$ [42], respectively, are not observed. Notice that, unlike most semiconductors, the optical absorption edge of $\mathrm{HgCdTe}$ shifts to high energies at elevated temperatures [42]. 


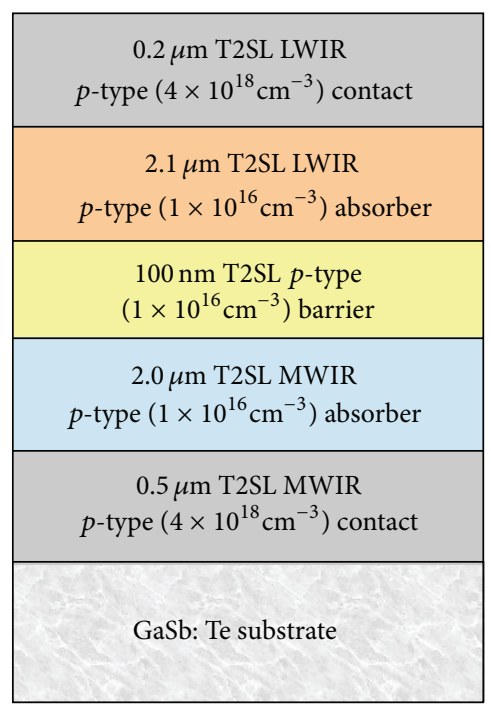

(a)

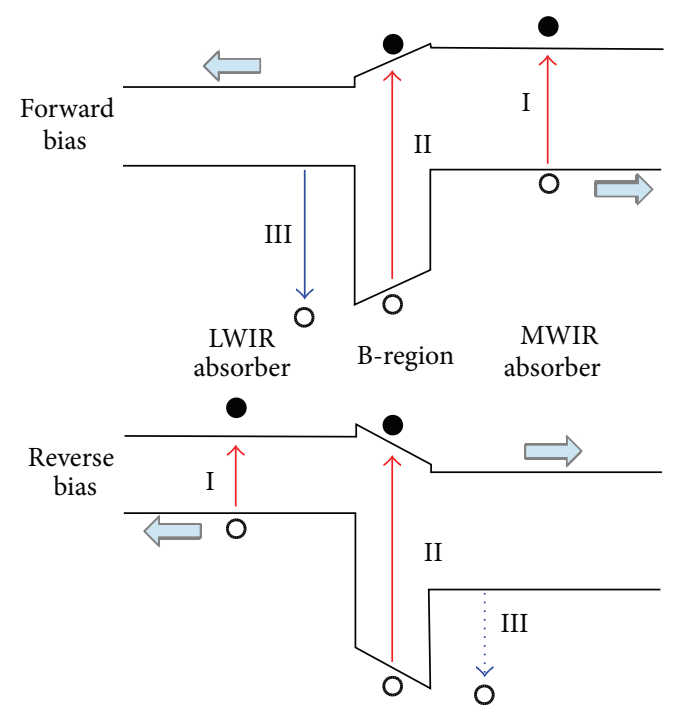

(b)

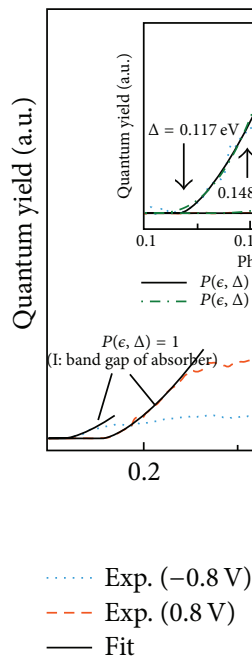

(c)

Figure 5: (a) Schematic of a pBp type-II InAs/GaSb superlattice photodetector. (b) Band alignment under forward and reverse biases and the effective optical transitions that contribute to photocurrents. (c) The experimental quantum yield spectra and IP fittings. The thresholds of optical transitions I-III can be obtained by fitting different energy regimes. Inset: comparison of fittings using different transmission probabilities.

Previous studies have shown that the assumption of the parabolic-band approximation (PBA), that is, JDOS $(\epsilon-h \nu)^{1 / 2}$, is valid for GaAs/AlGaAs heterojunctions [2] and type-II InAs/GaSb superlattice structures [8]. In these studies, dopant-caused band tailing has the negligible effects on the spectral yield. This is in part due to the process where the escape of photocarriers over a potential barrier [2] requires optical transitions ending up at the states with the energies greater than the barrier. In addition, the effect of the band tailing is insignificant for the GaAs and type-II superlattice absorber. For HgCdTe, however, the Urbach tail [43] is known to significantly distort the absorption edge. As shown in the inset of Figure 6(c), the IP fitting based on PBA agrees well with the experiment for the high-energy range but fails to explain the spectral yield in the near-threshold regime. One possible reason could be due to the trivial influence of the potential barrier on the escape of photocarriers, which means that the spectral yield is mostly determined by the $\mathrm{Hg}_{0.78} \mathrm{Cd}_{0.22} \mathrm{Te}$ absorber. As a consequence, band tailing should be properly considered in order to fit the yield spectra (see the solid line in the inset of Figure 6(c)).

In terms of the structure (Figure 6(a)), electrons escaping into the $\mathrm{Hg}_{0.68} \mathrm{Cd}_{0.32} \mathrm{Te}$ layer under a positive bias need to overcome a barrier resulting from the conduction band offset (CBO) between $\mathrm{Hg}_{0.78} \mathrm{Cd}_{0.22} \mathrm{Te}$ and $\mathrm{Hg}_{0.68} \mathrm{Cd}_{0.32}$ Te junction. The IP threshold of this should be different under negative bias where surmounting the barrier is not needed. However, such a difference cannot be identified from the spectral yield 


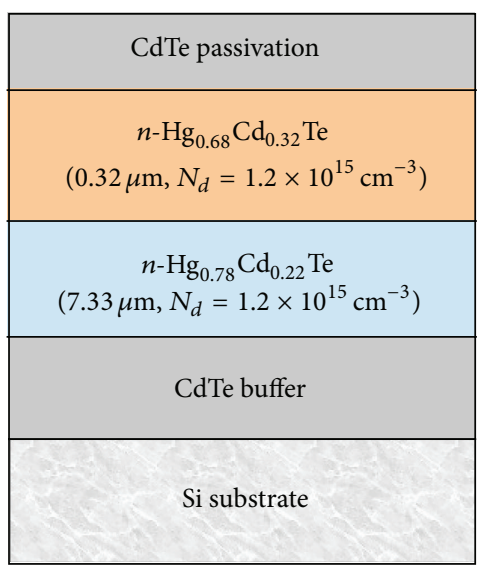

(a)



(b)



- - Exp. $(5.3 \mathrm{~K})$
$\ldots .$. Exp. $(78 \mathrm{~K})$
- Fit

(c)

Figure 6: (a) Schematic of the $n$-type $\mathrm{Hg}_{0.78} \mathrm{Cd}_{0.22} \mathrm{Te} / \mathrm{Hg}_{0.68} \mathrm{Cd}_{0.32}$ Te heterojunction structure. (b) The quantum yield spectra at 5.3 and $78 \mathrm{~K}$. Spectra measured at positive and negative biases have a similar profile. Inset shows the schematic band alight (without considering the space charge effect and composition grading at the junction interface) and the dominant optical transition which occurs in $\mathrm{Hg}_{0.78} \mathrm{Cd} \mathrm{d}_{0.22} \mathrm{Te}$. (c) $\mathrm{The}$ quantum yield spectra at $0.6 \mathrm{~V}$, along with IP fittings (solid line). Inset: comparison of fittings using different energy distribution functions, where $\rho_{1}$ is an exponential function, while $\rho_{2}$ represents PBA approximation.

as shown in Figure 6(b). Both under positive and negative biases display similar spectral profile, and no apparent shifting in the thresholds can be observed. This confirms the negligible electron barrier at the $n$-type $\mathrm{Hg}_{0.68} \mathrm{Cd}_{0.32} \mathrm{Te}$ $(0.32 \mu \mathrm{m}) / \mathrm{Hg}_{0.78} \mathrm{Cd}_{0.22} \mathrm{Te}(7.33 \mu \mathrm{m})$ heterojunction interface.

\section{IP-Based Photodetectors}

3.1. Split-Off Band Heterojunction Infrared Detectors. The use of $p$-type split-off transition is promising to develop photodetectors avoiding operation under cryogenic cooling conditions. Uncooled $p-\mathrm{GaAs} / \mathrm{Al}_{x} \mathrm{Ga}_{1-x}$ As split-off detectors with highly doped emitters were demonstrated operating through the IP mode [11]. The split-off transition corresponds to hole transitions between the light/heavy-hole bands $(\mathrm{LH} / \mathrm{HH})$ and the spin-orbit split-off (SO) band [11, 44]. The transition energy and spectral response range thus depend on the SO$\mathrm{HH}$ splitting energy. The photocarriers then escape over the AlGaAs barrier through an internal photoemission process. The room-temperature response [11] of a split-off detector (SP003) is shown in Figure 7(a). This detector contains 30 periods of $3 \times 10^{18} \mathrm{~cm}^{-3} p$-doped $18.8 \mathrm{~nm} \mathrm{GaAs}$ and $60 \mathrm{~nm}$ undoped $\mathrm{Al}_{0.57} \mathrm{Ga}_{0.47}$ As. The active region is sandwiched between two $p$-type GaAs layers doped to $1 \times 10^{19} \mathrm{~cm}^{-3}$. The obtained responsivity is $0.29 \mathrm{~mA} / \mathrm{W}$, and the corresponding detectivity $D^{*}$ is $6.8 \times 10^{5}$ Jones at $2.5 \mu \mathrm{m}$ and at room temperature. 


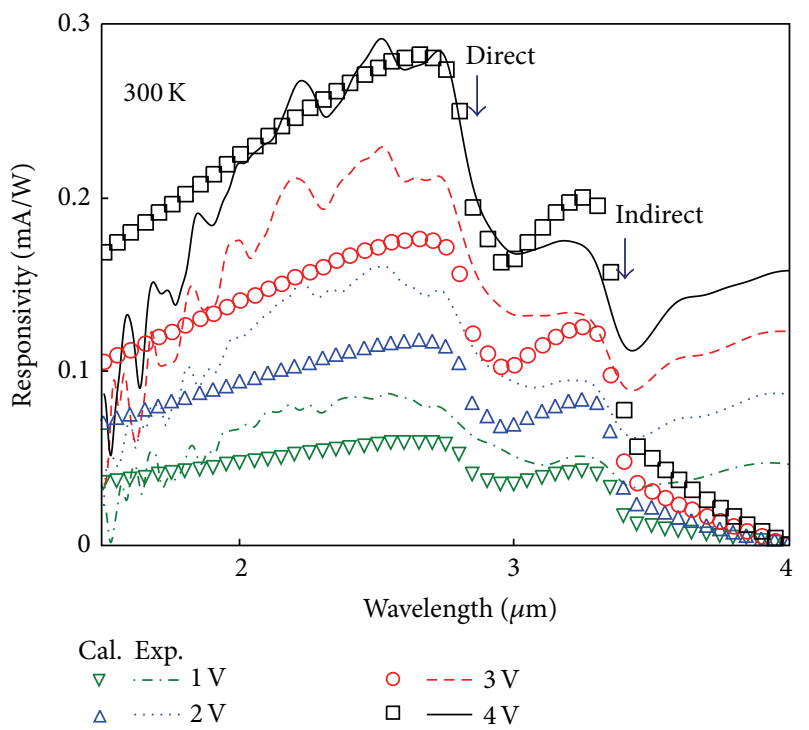

(a)

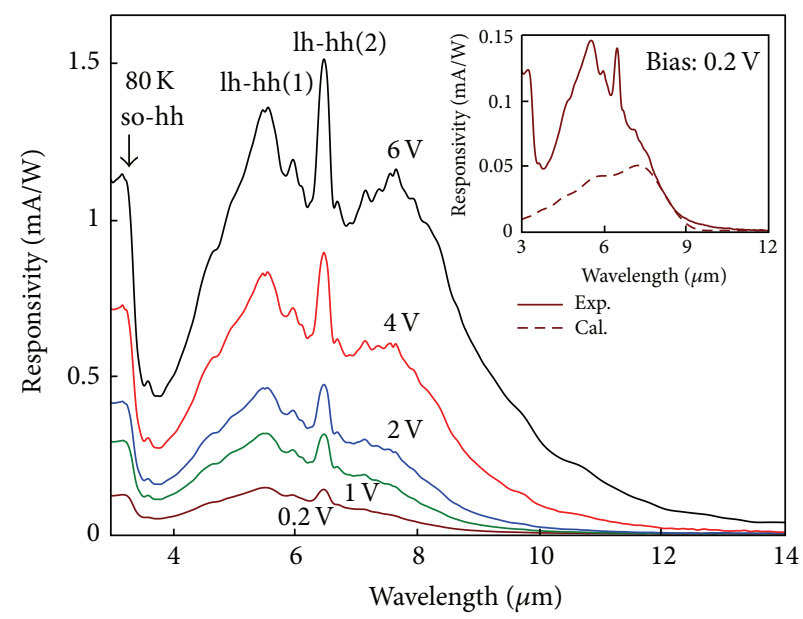

(b)

Figure 7: (a) The experimental and model response [10] for detector sample SP003 at different biases and $300 \mathrm{~K}$, due to the hole transition from heavy-hole (hh) to spin-orbit split-off (so) bands. Both direct $(\sim 2.7 \mu \mathrm{m})$ and indirect $(\sim 3.25 \mu \mathrm{m})$ transition peaks were observed. (b) The $80 \mathrm{~K}$ responsivity spectra of SP001 at different biases. The light-hole (lh) and heavy-hole (hh) transition contribute to the long-wavelength region $(>4 \mu \mathrm{m})$. A comparison of experiment with model free-carrier indirect responsivity (dashed line) for bias at $0.2 \mathrm{~V}$ is shown in the inset, indicating the lh-hh response superimposed on the free-carrier response.

In addition to the above split-off mechanism, the LH-HH transition is the main contribution to the long-wavelength spectral response $(4-10 \mu \mathrm{m})$. This is observed on a $p$ $\mathrm{GaAs} / \mathrm{Al}_{0.28} \mathrm{Ga}_{0.72}$ As heterostructure (SP001) [12] as shown in Figure 7(b), demonstrating the response up to $16.5 \mu \mathrm{m}$ at temperatures up to $330 \mathrm{~K}$ [12].

\subsection{Wavelength-Extended Photovoltaic Infrared Photode-} tectors. Photovoltaic detectors are attractive for achieving (i) extremely low noise, (ii) high impedance, and (iii) low power dissipation, compared to photoconductive detectors [45]. Various device concepts based on $p$ - $n$ junctions [46], quantum well (QW) [47], quantum dot (QD) [48, 49], typeII InAs/GaSb [50], and quantum cascade (QCD) structures $[49,51]$ have been explored to implement photovoltaic operation. One of the key factors is to have a built-in potential to sweep out the photocarriers without using an external electric field. Schneider et al. [47] reported a four-zone low noise photovoltaic QW infrared photodetector (QWIP) with the preferable transport of carriers toward one direction, while internal electric field is associated with the junction for a $n-i-p$ type-II InAs/GaSb/AlSb detector [50]. Recently, Barve and Krishna [49] reported a photovoltaic QD infrared photodetector (QDIP) based on the QCD concept [51], in which directional transfer of carriers between QDs is favored by cascade transitions through phonon coupling.

The present PV detector architecture uses the standard structure of internal photoemission detectors [12], in which a highly $p$-type doped GaAs acts as the photon absorber and emitter. Figure 8(a) shows the valence band, which has a nonsymmetrical band configuration. In addition to an offset $\left(\delta E_{v}\right)$ between the barriers below and above the emitter, one of the AlGaAs barriers is graded to further increase the nonsymmetry and facilitate the transport of photoexcited holes at zero bias. The room-temperature photovoltaic operation has been reported elsewhere [52]. For comparison, different gradients of $\mathrm{Al}$ fractions (i.e., $x$ varies from 0.45 to 0.75 or remains a constant (Table 2)) were investigated. Figure $8(\mathrm{~b})$ schematically shows the intervalence band (IVB) transitions mainly responsible for photon absorption and hole escape over the barrier (through internal photoemission). To determine $E_{A}$, the characteristics of dark current-voltagetemperature $(I-V-T)$ were measured (Figure $9(\mathrm{a})) . R_{0} A$ values extracted from $I-V-T$ data are shown in Figure 9(b), where a previous symmetrical $\mathrm{GaAs} / \mathrm{Al}_{0.57} \mathrm{Ga}_{0.43}$ As detector (sample SP3) [11] is also shown for comparison. The Arrhenius plots are used to fit $R_{0} A$ and determine $E_{A}$ to be 0.37 , 0.40 , and $0.49 \mathrm{eV}$ for samples SP1005, SP1007, and SP1001, respectively. Except for SP1001, the obtained $E_{A}$ values are comparable to the designed internal work function $(\Delta)$ of $\sim 0.40 \mathrm{eV}$, in accordance with the $p$-type $\mathrm{GaAs} / \mathrm{Al}_{0.75} \mathrm{Ga}_{0.25} \mathrm{As}$ junction by taking into account $p$-type-doping effects [2]. The $R_{0} A$ - $T$ characteristic giving $E_{A} \sim 0.4 \mathrm{eV}$ demonstrated that both SP1005 and SP1007 behave like a $3 \mu \mathrm{m}$ threshold detector.

The figure of merits for the photovoltaic operation is shown in Figures 10(a) and 10(b). $D^{*}$ was obtained [50] using the formula $D^{*}=\mathscr{R} /\left(2 q J+4 k T / R_{\text {diff }} A\right)^{1 / 2}$, in which $\mathscr{R}$ is the responsivity, $J$ is the dark current density, and $R_{\text {diff }}$ is the differential resistance. At zero bias where the shot noise vanishes, this expression is reduced to the normal form in terms of the Johnson noise [48]. With decreasing 
TABLE 2: GaAs/ $\mathrm{Al}_{x} \mathrm{Ga}_{1-x}$ As photovoltaic detector parameters. The active region consists of a $400 \mathrm{~nm}$ thick undoped $\mathrm{Al}_{x_{3}} \mathrm{Ga}_{1-x_{3}} \mathrm{As}$ barrier with constant $x_{3}$, a $p$-type GaAs layer (emitter) doped to $1 \times 10^{19} \mathrm{~cm}^{-3}$, and a flat (SP1001) or graded (SP1005 and SP1007) $80 \mathrm{~nm}$ thick $\mathrm{Al}_{x} \mathrm{Ga}_{1-x}$ As barrier ( $x$ varies from $x_{2}$ to $x_{1}$ ). Figure 8 (a) shows the valence band diagram. $d_{e}, E_{A}$, and $\lambda_{T}$ denote the thickness of emitter, activation energy, and the threshold wavelength of photovoltaic response, respectively.

\begin{tabular}{lcccccc}
\hline Sample number & $x_{1}$ & $x_{2}$ & $x_{3}$ & $d_{e}(\mathrm{~nm})$ & $E_{A}(\mathrm{eV})$ & 0.49 \\
\hline SP1001 (flat-barrier) & 0.75 & 0.75 & 0.57 & 80 & 20 & 0.37 \\
SP1005 (graded-barrier) & 0.45 & 0.75 & 0.57 & 8.9 & 8.0 \\
SP1007 (graded-barrier) & 0.45 & 0.75 & 0.57 & 80 & 0.40 & 8.0 \\
\hline
\end{tabular}

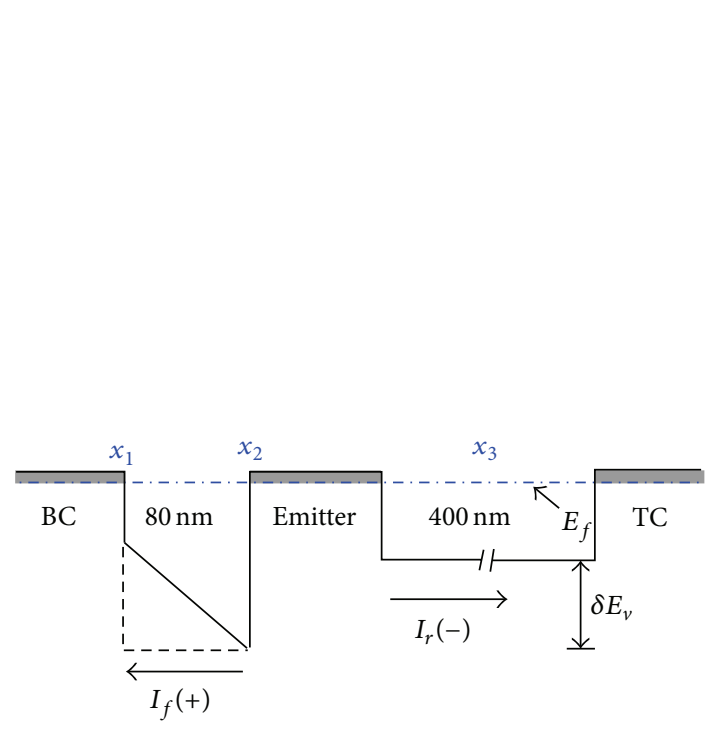

(a)



(b)

FIGURE 8: (a) The valence band diagram of a graded (solid line)/flat (dashed line) barrier structure. Bidirectional photocurrents can simultaneously exist in the sample for photovoltaic operation, as shown by $I_{f}(+)$ and $I_{r}(-)$, corresponding to forward and reverse photocurrents, respectively. $x_{1}, x_{2}$, and $x_{3}$ are the Al fractions of the barriers as described in Table 2. $\delta E_{v}$ is an offset between the barriers below and above the emitter. (b) Schematic of optical transitions between two valence bands, that is, the heavy-hole (hh) and light-hole (lh) bands. For holes to escape over a potential barrier, optical transitions typically occur with the assistance of phonons.

temperature, $R_{\text {diff }}$ rapidly increases for the bias around $0 \mathrm{~V}$. To calculate photovoltaic $D^{*}$ at $80 \mathrm{~K}$, extrapolation of $R_{0}$ in terms of the Arrhenius plots (Figure 9(b)) has been carried out.

The spectral response of the graded-barrier samples contains a feature, that is, the large redshift in the threshold wavelength under photovoltaic operation (doubling the operating wavelength range). From the photoresponse characteristic point of view, the device with $E_{A} \sim 0.40 \mathrm{eV}$ acts as an $8 \mu \mathrm{m}$ threshold detector. Notice that the flat-barrier sample SP1001 does not respond beyond $3.9 \mu \mathrm{m}$, which is similar to the symmetrical GaAs/ $\mathrm{Al}_{0.57} \mathrm{Ga}_{0.43}$ As detector as observed before (sample SP3) [11]. Another feature being observed is the zero-responsivity point lying between 3.4 and $3.5 \mu \mathrm{m}$, which is an indication of bidirectional photocurrents simultaneously existing in the sample (see Figure 8(a)). This can be understood since photoexcited holes in the emitter can emit over both sides of the barriers, which normally have the threshold wavelengths of 3 and $4 \mu \mathrm{ms}$, corresponding to the heterointerfaces of $\mathrm{GaAs} / \mathrm{Al}_{0.75} \mathrm{Ga}_{0.25}$ As and $\mathrm{GaAs} / \mathrm{Al}_{0.57} \mathrm{Ga}_{0.43} \mathrm{As}$, respectively. The photocurrent cancellation leads to the occurrence of zero response between 3 and $4 \mu \mathrm{ms}$. Several zero-response points taking place in sample SP1001 are related to its band alignment.

In general, threshold redshifting mostly results from biasrelated effects, such as image-force barrier lowering and quantum tunneling. However, these effects are absent in the photovoltaic operating mode. A testimony of our observations was justified as being due to the high-energy photon excitation. Since the bottom contact (BC), emitter, and top contact (TC) are highly doped, photoexcited holes can be created in all of these layers. Because of the graded-barrier, high-energy photons can give rise to a net flow of photoexcited holes from BC to emitter. Some of the photoexcited holes are captured by the emitter. These high-energy captured holes tend to increase the energies of holes [10] originally in the emitter and excite them to high-energy states. As a consequence, the energies of photons needed in order to 


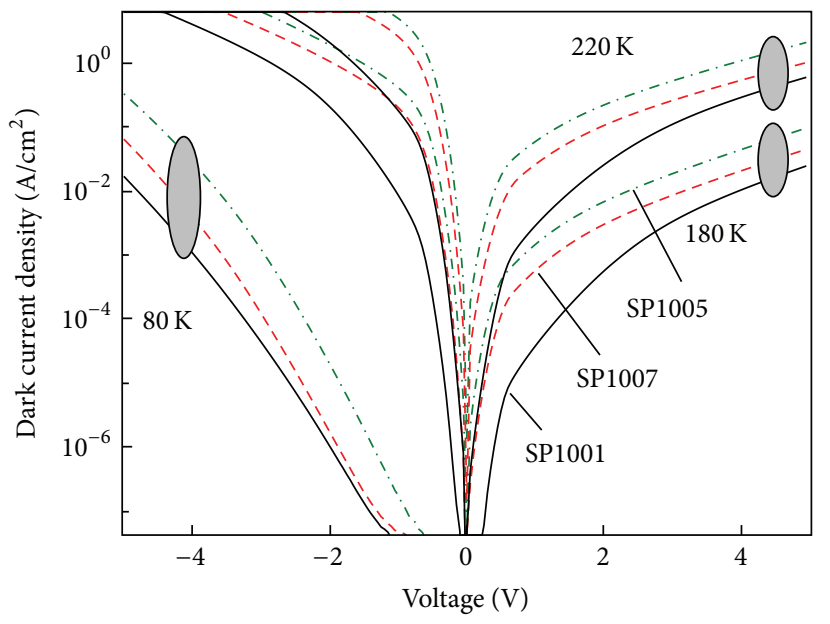

(a)

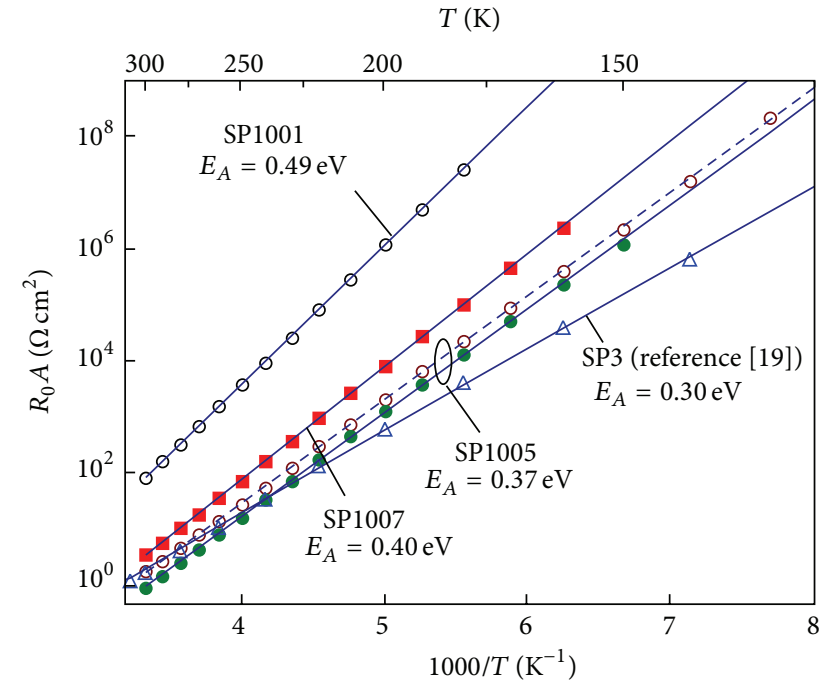

(b)

Figure 9: (a) Dark $I-V$ characteristics at different temperatures. (b) The Arrhenius plots of $R_{0} A$ against 1000/T, to determine the activation energies $\left(E_{A}\right)$. All of data result from measurements of $400 \times 400 \mu \mathrm{m}^{2}$ mesas, except for SP1005, for which an additional device with the dimension of $800 \times 800 \mu \mathrm{m}^{2}$ (open circles) is plotted to confirm the consistency. Also shown is a previously reported [11] symmetrical flatbarrier detector (SP3), which has the zero barrier offset; that is, $\delta E_{v}=0 \mathrm{eV}$. Its $E_{A}$ corresponds to the activation energy of the $p$-type GaAs $\left(3 \times 10^{18} \mathrm{~cm}^{-3}\right) / \mathrm{Al}_{0.57} \mathrm{Ga}_{0.43}$ As junction.

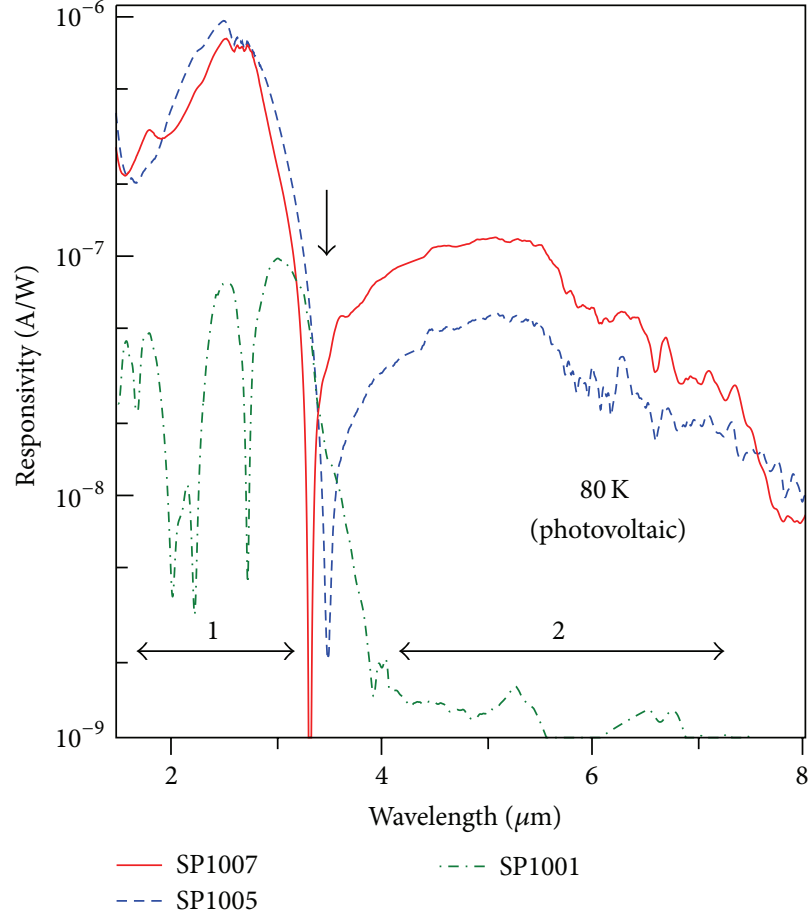

(a)



(b)

Figure 10: The spectral (a) responsivity and (b) detectivity at $80 \mathrm{~K}$. The vertical arrows indicate the occurrence of zero response due to bidirectional photocurrents simultaneously existing in the device, owing to the photovoltaic operating mode. Labeled responses (1) and (2) correspond to the forward $\left[I_{f}(+)\right]$ and reverse $\left[I_{r}(-)\right]$ photocurrents. Long-wavelength response beyond $3.9 \mu \mathrm{m}$ was not observed in flatbarrier sample SP1001. Table 2 summarizes the threshold wavelengths of photovoltaic response for three samples. The $D^{*}$ value of SP1007 is $10^{5}$ times higher than 30-period photoconductive detectors previously reported [12]. 




(a)

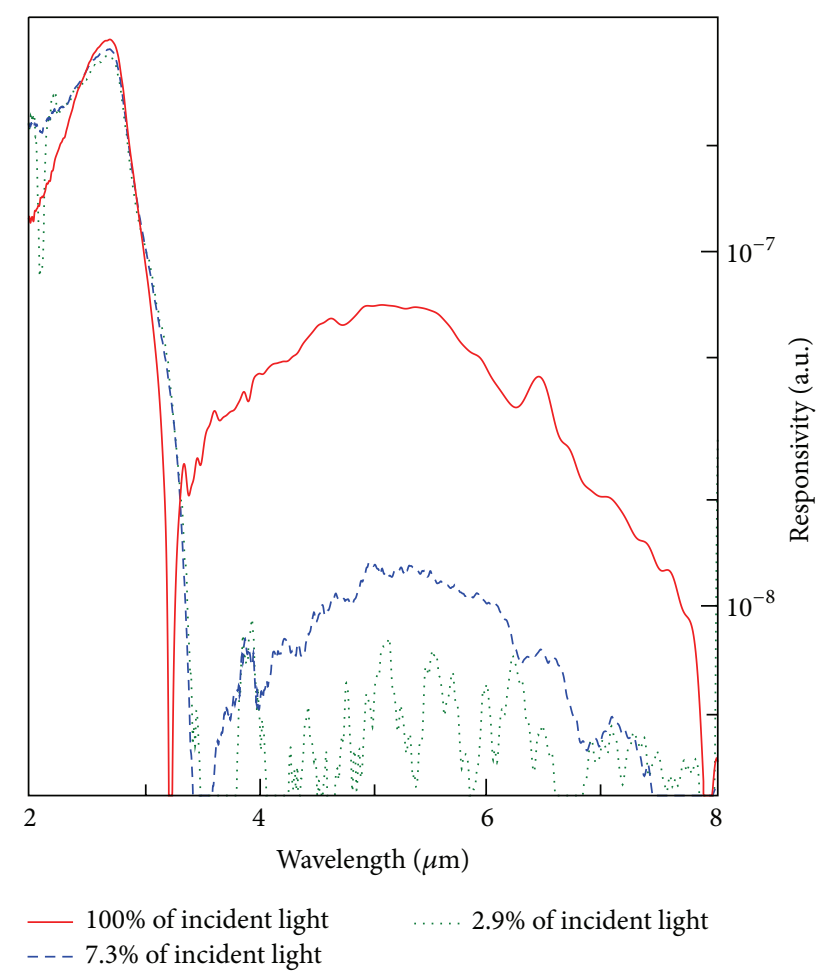

(b)

Figure 11: (a) Photovoltaic response measured by using different long-pass filters ( $\lambda_{\mathrm{CO}}$ is the cut-on wavelength). A filter with $\lambda_{\mathrm{CO}}=3.60 \mu \mathrm{m}$ blocks the transport of photoexcited holes overcoming the graded-barrier region, thus suppressing both the short- and long-wavelength response. (b) Variation of photovoltaic response with the intensities of incident light (through adjusting the optical aperture size of the spectrometer), where $100 \%$ of light corresponds to the default aperture size. The calibration of responsivity takes into account the variation of light, which leads to nearly unchanged responsivity values for the $<3 \mu \mathrm{m}$ wavelength range. The $>3.5 \mu \mathrm{m}$ response becomes almost negligible when the intensity of incoming light is reduced to $2.9 \%$.

excite holes to escape over the barrier can be correspondingly reduced, giving rise to a long-wavelength response. This process takes place in the hole transport from $\mathrm{BC}$ to $\mathrm{TC}$, in agreement with observed wavelength-extended response corresponding to the reverse photocurrents (Figure 10).

To justify the above mechanism, spectral response has been measured with the use of different long-pass filters (with the cut-on wavelength of $\lambda_{\mathrm{CO}}$ ) and different intensities of incoming light, as shown in Figure 11. This varies the energy or the concentration of photoexcited holes injected into the emitter. By using a filter with $\lambda_{\mathrm{CO}}=3.60 \mu \mathrm{m}$, the short-wavelength response (labeled region 1 of Figure 10) should disappear, as the escape of holes from the emitter to the $\mathrm{BC}$ cannot be accomplished because of the missing of the $h \nu>0.34 \mathrm{eV}(\lambda<3.60 \mu \mathrm{m})$ photons. For the same reason, photoexcited holes in the $\mathrm{BC}$ will be unable to overcome the graded-barrier (highest barrier $\sim 0.4 \mathrm{eV}$ ) to enter into emitter. This suppresses the long-wavelength response as well (labeled region 2 of Figure 10), according to the aforementioned energy transfer mechanism. As expected (Figure 11(a)), photovoltaic response was unseen throughout the entire spectral range. In contrast, the use of a filter with $\lambda_{\mathrm{CO}}=2.40 \mu \mathrm{m}$ gives rise to both short- and long-wavelength response owing to allowed emitter-to-BC and $\mathrm{BC}$-to-emitter hole transport. The efficiency of energy transfer between photoexcited holes and holes in the emitter could be a critical factor determining the long-wavelength response. Such energy transfer results from carrier scatterings and could be subject to degradation from hole-impurity scattering as the emitter is highly doped. With increasing the concentration of photoexcited holes, enhanced hole-hole scatterings can be expected, leading to distinct response in long-wavelength range (Figure 11(b)). By taking into account the intensity of the incoming light, the short-wavelength portion of the response is expected to remain the same. However, the long-wavelength portion is almost negligible when the light intensity is reduced to $2.9 \%$ and quickly rises up when the incoming light increases to $7.3 \%$, where $100 \%$ of light corresponds to the default optical aperture in the experiment. This may also explain the response characteristic of sample SP1001 not beyond $3.9 \mu \mathrm{m}$, where because of the flat-barrier configuration the net injection of photoexcited holes from the $\mathrm{BC}$ to emitter is negligible.

Higher activation energy of the dark current-voltage characteristics than the photoresponse threshold energy can provide a significant improvement of the detector performance. According to $R_{0} A \sim \exp \left(-E_{A} / k T\right)$, the $R_{0} A$ value (at $80 \mathrm{~K}$ ) of our detector with $E_{A}=0.40 \mathrm{eV}$ (responding up to 




(a)

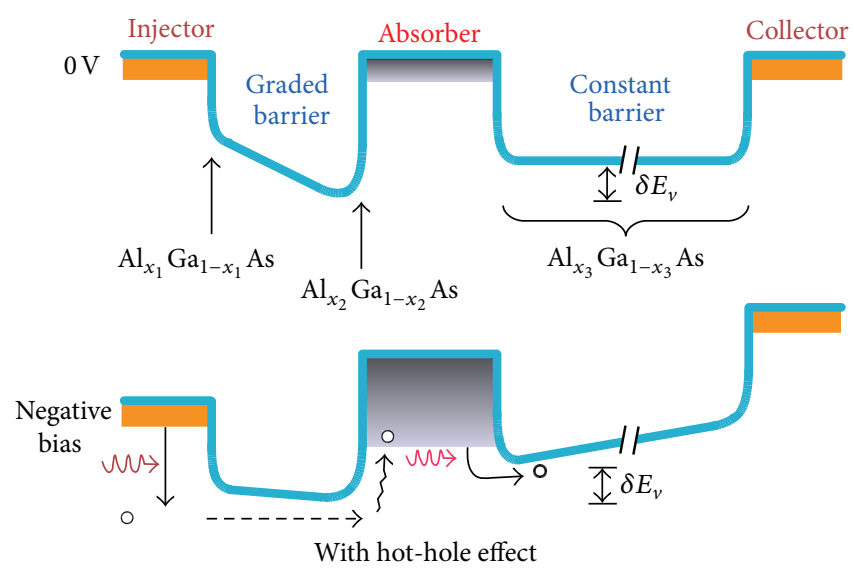

(b)

Figure 12: (a) The structure of the $p$-type GaAs/Al $\mathrm{Ga}_{1-x}$ As hot-hole photodetector. Graded-barrier Al fractions vary from $x_{1}$ to $x_{2}$, while the constant barrier $\mathrm{Al}$ fraction is $x_{3}$. (b) Schematic of the valence band alignment (including band bending). The offset between the barriers below and above the $p$-GaAs absorber is labeled as $\delta E_{v}$.

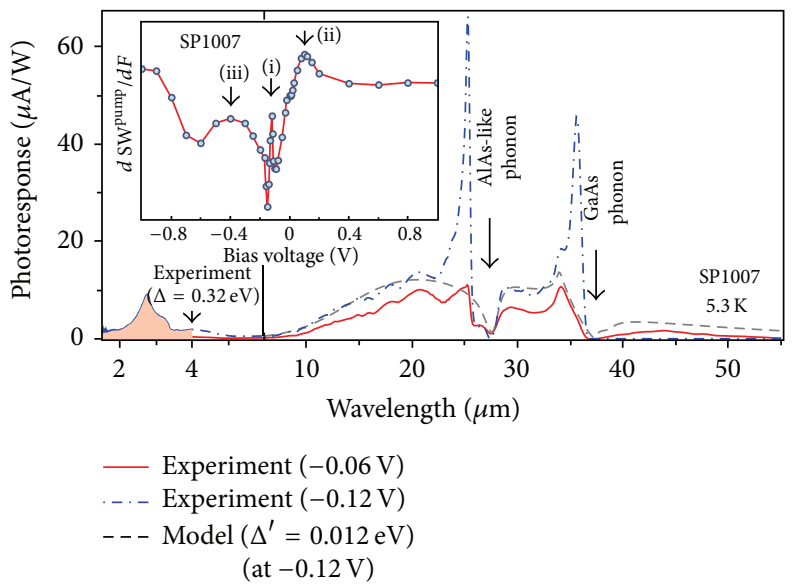

Figure 13: Measured photoresponse at $5.3 \mathrm{~K}$, and the fittings based on the escape-cone model. The fittings give a reduced threshold energy at $\Delta^{\prime}=0.012 \mathrm{eV}$. The vertical arrows indicate the GaAs and AlAs-like phonons. Inset: differential SW ${ }^{\text {pump }}$.

$8 \mu \mathrm{m})$ is nearly $10^{15}$ times higher than a conventional detector with $E_{A}$ of $0.155 \mathrm{eV}$ (without wavelength extension, also responding up to $8 \mu \mathrm{m}$ ), which gives nearly $10^{7}$ improvement in $D^{*}$. To experimentally evaluate the $D^{*}$ improvement factor, same type of internal photoemission detectors [12] was compared, as shown in Figure 10(b) (this detector contains 30 periods of emitters and barriers), which is nearly $10^{5}$ times less than the present detector.

\subsection{Hot-Hole Photodetectors with a Response Beyond the Band} Gap Spectral Limit. A novel concept of photodetection is developed based on an energy transfer process between hot and cold carriers, enabling spectral extension in surpassing the standard limit set by $\lambda_{c}=h c / \Delta[14]\left(\lambda_{c}\right.$ is the threshold wavelength and $\Delta$ is the activation energy). The principle is associated with the excitation of cold holes into higher energy states by interacting with hot holes and thus the responding of these high-energy carriers to the long-wavelength infrared radiation.

The $p$-type samples $\mathrm{GaAs} / \mathrm{Al}_{x} \mathrm{Ga}_{1-x}$ As were used to demonstrate hot-hole response. As shown in Figure 12(a), these samples contain $p$-type doped $\left(1 \times 10^{19} \mathrm{~cm}^{-3}\right) \mathrm{GaAs}$ layers; they are the injector, absorber, and collector, respectively. The VB alignment is schematically plotted in Figure 12(b). By absorbing photons, the holes in the injector are excited and injected into the absorber. After passing over the barrier, these holes have high energies and thus are "hot" compared to the original holes in the absorber [53].

Photoresponse was measured at $5.3 \mathrm{~K}$ as plotted in Figure 13, which shows a very long-wavelength infrared 


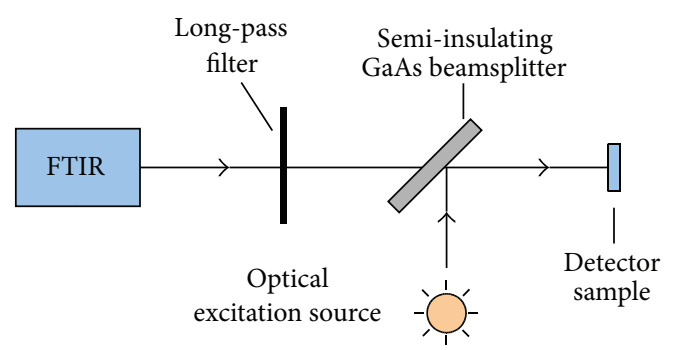

FIGURE 14: Experimental apparatus, where the double-side polished semi-insulating GaAs wafer acts as a beamsplitter.

(VLWIR) response. In terms of the internal work function (i.e., $\Delta)$ [2] of the absorber/constant barrier junction $(\Delta=$ $0.32 \mathrm{eV}$ ), the designed limit should be only $3.9 \mu \mathrm{m}$ (shaded region). As shown in Figure 13, the VLWIR response can be explained by a fitting [54] using the value $\Delta=0.012 \mathrm{eV}$.

The hot-cold carrier energy transfer mechanism is in part supported by the differential photocurrent. The shortwavelength light in the measurement (e.g., from the Fourier transform infrared (FTIR) spectrometer) is denoted as the "pump." The pump light is responsible for the generation of hot holes, which can be described using a three-dimensional drift model [45],

$$
I_{\mathrm{ph}}^{\mathrm{pu}}=e \cdot v(F) \int_{\Delta}^{+\infty} N(\epsilon) d \epsilon,
$$

where $I_{\mathrm{ph}}^{\mathrm{pu}}$ represents the pump current and $N(\epsilon)$ is the hole concentration [55]. Taking the derivative of $I_{\mathrm{ph}}^{\mathrm{pu}}$ with respect to $F$ gives

$$
\frac{d I_{\mathrm{ph}}^{\mathrm{pu}}}{d F}=e \cdot \frac{d v(F)}{d F} \int_{\Delta}^{+\infty} N(\epsilon) d \epsilon-e \cdot v(F) \frac{d \Delta}{d F} \cdot N(\Delta)
$$

in which the image-force barrier lowering and the tilting of the graded-barrier under the field have a primary effect on $d \Delta / d F$ [2]. For the high-field region, the first term of (7) is negligible. The differential $I_{\mathrm{ph}}^{\mathrm{pu}}$ thus determines the energy distribution of holes. $I_{\mathrm{ph}}^{\mathrm{pu}}$ can be evaluated as being proportional to the spectral weight (SW) of the response, defined as

$$
\mathrm{SW} \propto \int_{\lambda_{\min }}^{\lambda_{\max }} \mathscr{R}(\lambda) d \lambda
$$

where $\mathscr{R}(\lambda)$ is the spectral responsivity. The inset of Figure 10 shows three peaks at $-0.12,-0.40$, and $0.10 \mathrm{~V}$. As a comparison, another sample, which does not display the VLWIR response peaks, only displays one differential SW peak at $0 \mathrm{~V}$. The previous studies using hot-carrier spectroscopy $[55,56]$ indicate that the distribution peaks are the sign of the hotcold carrier interaction.

The VLWIR response as a consequence of the hotcold carrier energy transfer indicates that the response can be tuned by varying the degree of the hot-hole injection. Figure 14 plots the experimental setup. A long-pass filter with the cut-on wavelength $\lambda_{\mathrm{CO}}=4.5 \mu \mathrm{m}$ (corresponding to $0.28 \mathrm{eV})$ is used to block the high-energy $(>0.28 \mathrm{eV})$ photons (from spectrometer). The hot holes are provided by photoexcitation using an external high-energy light. Figure 15 shows the photocurrent (spectral weight of the response) at different excitation levels. The result indicates that increasing the excitation intensity enables the VLWIR response.

In summary, we presented IP studies on InAs/GaSb T2SL and HgCdTe photodetectors. The band gaps of photon absorbers and their temperature dependence are determined by IP spectroscopy, based on fittings to the quantum yield near-threshold regimes. We also demonstrated an IP detector with extended response up to $55 \mu \mathrm{m}$. The tunability of the response is demonstrated by varying the degree of the hothole injection.

\section{Outlook of the IP Methodology}

The generic characteristics of IP is associated with the process of generation and emission of photocarriers. IP exists in a variety of optical processes including the bulk [57], quantum [58], and even semiconductor-liquid electrolyte junctions [59]. The IP signal can be detected through an electric field [57], a second photon [60], or second-harmonic generation [61]. Therefore, IP can be applied to a large extent of materials and heterojunction. The variation in the way of detecting the signal will also enable studying different properties than those that have been reviewed in this paper, for example, the carrier-carrier/phonon scatterings.

This review has focused on the IP phenomena in the infrared wavelength range which typically corresponds to the energy of photon less than the value of the band gap. A more comprehensive review of IP on studying optical process across the band gap and in the wide-band gap materials can be found in [1].

\section{Conflict of Interests}

The authors declare that there is no conflict of interests regarding the publication of this paper.

\section{Acknowledgments}

This work was supported in part by the US Army Research Office under Grant no. W911NF-15-1-0018 monitored by Dr. William W. Clark and in part by the US National Science Foundation under Grant no. ECCS-1232184. 

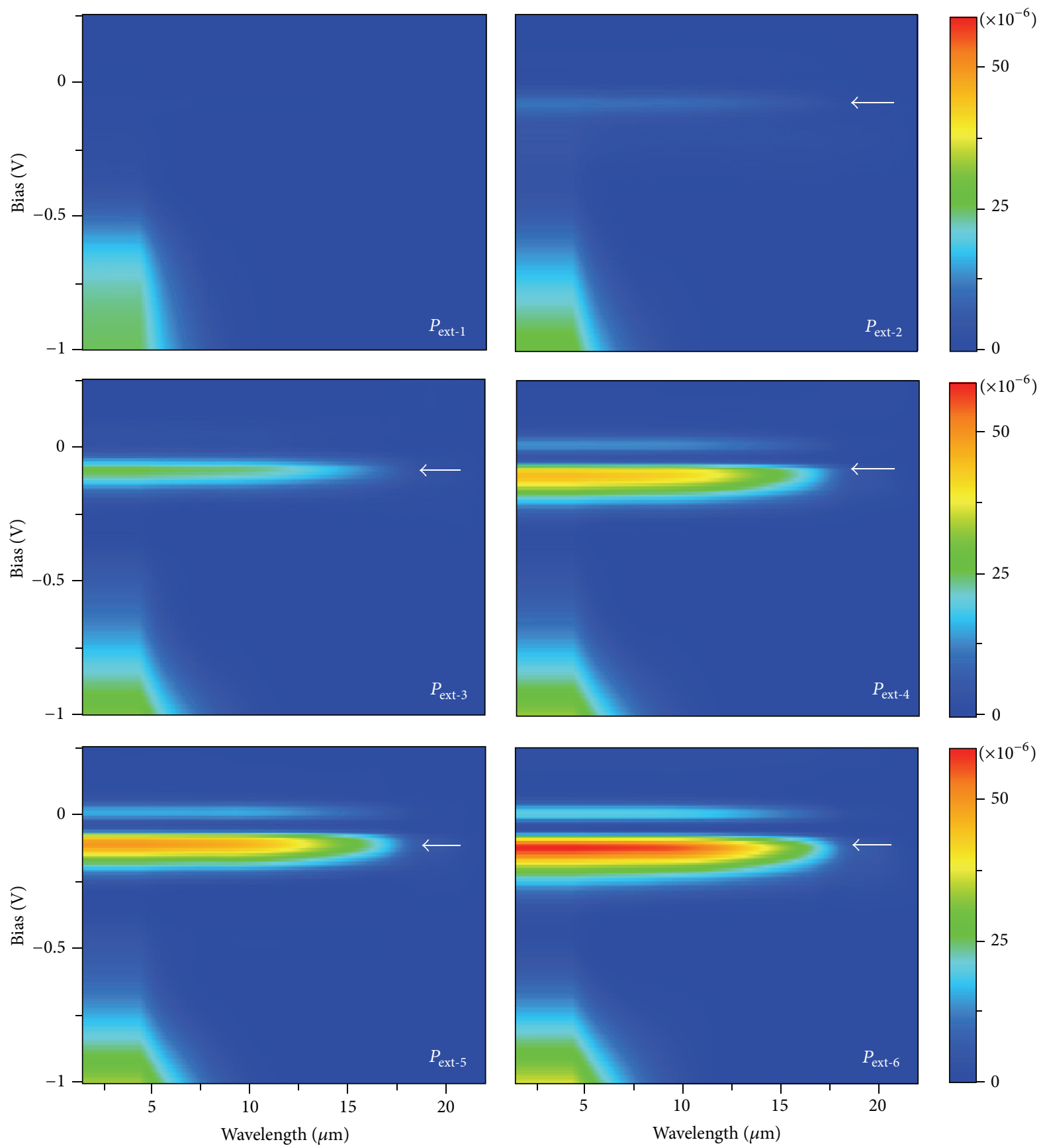

(a)

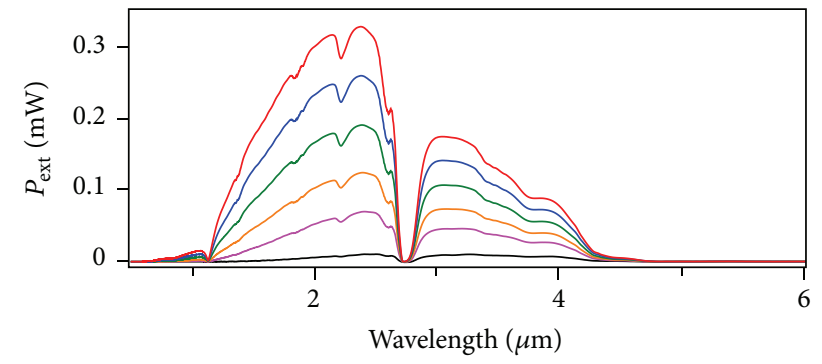

$$
\begin{array}{ll}
-P_{\text {ext-6 }} & -P_{\text {ext-3 }} \\
-P_{\text {ext-5 }} & -P_{\text {ext-2 }} \\
-P_{\text {ext-4 }} & -P_{\text {ext-1 }}
\end{array}
$$

(b)

FIgURE 15: Continued. 




(d)

FIGURE 15: (a) Spectral weights of response. Different excitation levels are indicated as $P_{\text {ext- } 1}-P_{\text {ext-8 }}$. The sample under measurements is SP1006. (b) Power of the external excitation optical source (incident on the active area of $260 \times 260 \mu \mathrm{m}^{2}$ ). (c)-(d) The VLWIR response (at $-0.1 \mathrm{~V}$ ) varying with the excitation power. The arrows indicate the cut-on wavelength of the filter and the $2 \times \mathrm{TO}(X)$ phonon features of the GaAs beamsplitter, respectively.

\section{References}

[1] V. Afanas'ev, Internal Photoemission Spectroscopy: Principles and Applications, Elsevier Science, 2010.

[2] Y.-F. Lao and A. G. U. Perera, "Temperature-dependent internal photoemission probe for band parameters," Physical Review B, vol. 86, no. 19, Article ID 195315, 9 pages, 2012.

[3] R. Yan, Q. Zhang, W. Li et al., "Determination of graphene work function and graphene-insulator-semiconductor band alignment by internal photoemission spectroscopy," Applied Physics Letters, vol. 101, no. 2, Article ID 022105, 2012.

[4] Y. Hikita, M. Kawamura, C. Bell, and H. Y. Hwang, "Electric field penetration in $\mathrm{Au} / \mathrm{Nb}: \mathrm{SrTiO}_{3}$ Schottky junctions probed by bias-dependent internal photoemission," Applied Physics Letters, vol. 98, no. 19, Article ID 192103, 2011.

[5] J. Batey and S. L. Wright, "Energy band alignment in GaAs:(Al,Ga)As heterostructures: the dependence on alloy composition," Journal of Applied Physics, vol. 59, no. 1, pp. 200209, 1986.

[6] T. B. Bahder, "Eight-band k.p model of strained zinc-blende crystals," Physical Review B, vol. 41, no. 17, pp. 11992-12001, 1990.

[7] P. Lautenschlager, M. Garriga, S. Logothetidis, and M. Cardona, "Interband critical points of GaAs and their temperature dependence," Physical Review B, vol. 35, no. 17, pp. 9174-9189, 1987.
[8] Y.-F. Lao, P. K. D. D. P. Pitigala, A. G. Unil Perera, E. Plis, S. S. Krishna, and P. S. Wijewarnasuriya, "Band offsets and carrier dynamics of type-II InAs/GaSb superlattice photodetectors studied by internal photoemission spectroscopy," Applied Physics Letters, vol. 103, no. 18, Article ID 181110, 2013.

[9] Y.-F. Lao, A. G. U. Perera, L. H. Li, S. P. Khanna, E. H. Linfield, and H. C. Liu, "Tunable hot-carrier photodetection beyond the bandgap spectral limit," Nature Photonics, vol. 8, no. 5, pp. 412418, 2014.

[10] S. G. Matsik, P. V. V. Jayaweera, A. G. U. Perera, K. K. Choi, and P. Wijewarnasuriya, "Device modeling for split-off band detectors," Journal of Applied Physics, vol. 106, no. 6, Article ID 064503, 2009.

[11] P. V. V. Jayaweera, S. G. Matsik, A. G. U. Perera, H. C. Liu, M. Buchanan, and Z. R. Wasilewski, "Uncooled infrared detectors for 3-5 $\mu \mathrm{m}$ and beyond," Applied Physics Letters, vol. 93, no. 2, Article ID 021105, 2008.

[12] Y. F. Lao, P. K. D. D. P. Pitigala, A. G. U. Perera et al., "Lighthole and heavy-hole transitions for high-temperature longwavelength infrared detection," Applied Physics Letters, vol. 97, no. 9, Article ID 091104, 2010.

[13] R. H. Fowler, "The analysis of photoelectric sensitivity curves for clean metals at various temperatures," Physical Review, vol. 38 , no. 1 , pp. $45-56,1931$. 
[14] S. M. Sze and K. K. Ng, Physics of Semiconductor Devices, John Wiley \& Sons, New York, NY, USA, 2007.

[15] C. Coluzza, E. Tuncel, J.-L. Staehli et al., "Interface measurements of heterojunction band lineups with the Vanderbilt freeelectron laser," Physical Review B, vol. 46, no. 19, pp. 1283412836, 1992.

[16] C. Caroli, J. S. Helman, and F. S. Sinencio, "Internal photoemission of holes from a semiconductor into a semiconductor," Physical Review B, vol. 11, no. 2, article 980, 1975.

[17] E. O. Kane, "Theory of photoelectric emission from semiconductors," Physical Review, vol. 127, no. 1, pp. 131-141, 1962.

[18] W. Seidel, O. Krebs, P. Voisin, J. C. Harmand, F. Aristone, and J. F. Palmier, "Band discontinuities in $\mathrm{In}_{x} \mathrm{Ga}_{1-x}$ As-InP and InP$\mathrm{Al}_{y} \mathrm{In}_{1-y}$ as heterostructures: evidence of noncommutativity," Physical Review B, vol. 55, no. 4, pp. 2274-2279, 1997.

[19] V. V. Afanas'ev and A. Stesmans, "Internal photoemission at interfaces of high- $\kappa$ insulators with semiconductors and metals," Journal of Applied Physics, vol. 102, no. 8, Article ID 081301, 28 pages, 2007.

[20] R. Williams, "Injection phenomena," in Semiconductors and Semimetals, R. Willardson and A. Beer, Eds., chapter 2, Academic Press, New York, NY, USA, 1970.

[21] A. M. Goodman, "Photoemission of electrons from $n$-type degenerate silicon into silicon dioxide," Physical Review, vol. 152, no. 2, pp. 785-787, 1966.

[22] R. J. Powell, "Interface barrier energy determination from voltage dependence of photoinjected currents," Journal of Applied Physics, vol. 41, no. 6, pp. 2424-2432, 1970.

[23] E. O. Kane, "Thomas-fermi approach to impure semiconductor band structure," Physical Review, vol. 131, no. 1, pp. 79-88, 1963.

[24] J. Noffsinger, E. Kioupakis, C. G. Van de Walle, S. G. Louie, and M. L. Cohen, "Phonon-assisted optical absorption in silicon from first principles," Physical Review Letters, vol. 108, no. 16, Article ID 167402, 2012.

[25] A. Frova and P. Handler, "Direct observation of phonons in silicon by electric-field-modulated optical absorption," Physical Review Letters, vol. 14, no. 6, pp. 178-180, 1965.

[26] R. Newman and W. W. Tyler, "Effect of impurities on free-hole infrared absorption in p-type germanium," Physical Review, vol. 105, no. 3, pp. 885-886, 1957.

[27] R. Braunstein and L. Magid, "Optical absorption in p-type gallium arsenide," Physical Review, vol. 111, no. 2, pp. 480-481, 1958.

[28] M. Cardona, K. L. Shaklee, and F. H. Pollak, "Electroreflectance at a semiconductor-electrolyte interface," Physical Review, vol. 154, no. 3, article 696, 1967.

[29] M. Muñoz, K. Wei, F. H. Pollak, J. L. Freeouf, and G. W. Charache, "Spectral ellipsometry of GaSb: experiment and modeling," Physical Review B, vol. 60, no. 11, pp. 8105-8110, 1999.

[30] A. G. Thompson, M. Cardona, K. L. Shaklee, and J. C. Woolley, "Electroreflectance in the GaAs-GaP alloys," Physical Review, vol. 146, no. 2, pp. 601-610, 1966.

[31] I. Vurgaftman, J. R. Meyer, and L. R. Ram-Mohan, "Band parameters for III-V compound semiconductors and their alloys," Journal of Applied Physics, vol. 89, no. 11, pp. 5815-5875, 2001.

[32] J. S. Blakemore, "Semiconducting and other major properties of gallium arsenide," Journal of Applied Physics, vol. 53, no. 10, pp. R123-R181, 1982.

[33] E. O. Kane, "Energy band structure in p-type germanium and silicon," Journal of Physics and Chemistry of Solids, vol. 1, no. 1-2, pp. 82-99, 1956.
[34] M. Kuball, M. K. Kelly, M. Cardona, K. Köhler, and J. Wagner, "Doping dependence of the $E_{1}$ and $E_{1}+\Delta_{1}$ critical points in highly doped n- and p-type GaAs: importance of surface band bending and depletion," Physical Review B, vol. 49, no. 23, pp. 16569-16574, 1994.

[35] M. Heiblum, D. Galbi, and M. Weckwerth, "Observation of single-optical-phonon emission," Physical Review Letters, vol. 62, no. 9, article 1057, 1989.

[36] Q. O. Hu, E. S. Garlid, P. A. Crowell, and C. J. Palmstrøm, "Spin accumulation near Fe/GaAs (001) interfaces: the role of semiconductor band structure," Physical Review B, vol. 84, no. 8, Article ID 085306, 5 pages, 2011.

[37] D. C. Tsui, "Evidence for Hole-To-Phonon interaction from tunneling measurements in GaAs-Pb junctions," Physical Review Letters, vol. 21, no. 14, pp. 994-996, 1968.

[38] J. L. T. Waugh and G. Dolling, "Crystal dynamics of gallium arsenide," Physical Review, vol. 132, no. 6, pp. 2410-2412, 1963.

[39] P. Giannozzi, S. de Gironcoli, P. Pavone, and S. Baroni, “Ab initio calculation of phonon dispersions in semiconductors," Physical Review B, vol. 43, no. 9, pp. 7231-7242, 1991.

[40] S. Steiger, M. Salmani-Jelodar, D. Areshkin et al., "Enhanced valence force field model for the lattice properties of gallium arsenide," Physical Review B-Condensed Matter and Materials Physics, vol. 84, no. 15, Article ID 155204, 2011.

[41] E. A. Plis, S. S. Krishna, N. Gautam, S. Myers, and S. Krishna, "Bias switchable dual-band InAs/GaSb superlattice detector with pBp architecture," IEEE Photonics Journal, vol. 3, no. 2, pp. 234-240, 2011.

[42] J. P. Laurenti, J. Camassel, A. Bouhemadou, B. Toulouse, R. Legros, and A. Lusson, "Temperature dependence of the fundamental absorption edge of mercury cadmium telluride," Journal of Applied Physics, vol. 67, no. 10, pp. 6454-6460, 1990.

[43] F. Urbach, "The long-wavelength edge of photographic sensitivity and of the electronic absorption of solids," Physical Review, vol. 92 , no. 5, p. 1324, 1953.

[44] A. G. U. Perera, "Quantum structures for multiband photon detection," Opto-Electronics Review, vol. 14, no. 2, pp. 103-112, 2006.

[45] H. Schneider and H. C. Liu, Quantum Well Infrared Photodetectors: Physics and Applications, vol. 126 of Springer Series in Optical Sciences, Springer, New York, NY, USA, 2007.

[46] A. Rogalski, "Heterostructure infrared photovoltaic detectors," Infrared Physics \& Technology, vol. 41, no. 4, pp. 213-238, 2000.

[47] H. Schneider, C. Schönbein, M. Walther, K. Schwarz, J. Fleissner, and P. Koidl, "Photovoltaic quantum well infrared photodetectors: the four-zone scheme," Applied Physics Letters, vol. 71, no. 2, pp. 246-248, 1997.

[48] L. Nevou, V. Liverini, F. Castellano, A. Bismuto, and J. Faist, "Asymmetric heterostructure for photovoltaic InAs quantum dot infrared photodetector," Applied Physics Letters, vol. 97, no. 2, Article ID 023505, 2010.

[49] A. V. Barve and S. Krishna, "Photovoltaic quantum dot quantum cascade infrared photodetector," Applied Physics Letters, vol. 100, no. 2, p. 021105, 2012.

[50] A. M. Hoang, G. Chen, A. Haddadi, S. Abdollahi Pour, and M. Razeghi, "Demonstration of shortwavelength infrared photodiodes based on type-II InAs/GaSb/AlSb superlattices," Applied Physics Letters, vol. 100, no. 21, Article ID 211101, 2012.

[51] F. R. Giorgetta, E. Baumann, M. Graf et al., "Quantum cascade detectors," IEEE Journal of Quantum Electronics, vol. 45, no. 8, pp. 1039-1052, 2009. 
[52] P. K. D. D. P. Pitigala, S. G. Matsik, A. G. U. Perera et al., "Photovoltaic infrared detection with p-type graded barrier heterostructures," Journal of Applied Physics, vol. 111, no. 8, Article ID 084505, 2012.

[53] F. Capasso, "Band-gap engineering: from physics and materials to new semiconductor devices," Science, vol. 235, no. 4785, pp. 172-176, 1987.

[54] D. G. Esaev, M. B. M. Rinzan, S. G. Matsik, and A. G. U. Perera, "Design and optimization of GaAs/AlGaAs heterojunction infrared detectors," Journal of Applied Physics, vol. 96, no. 8, pp. 4588-4597, 2004.

[55] J. R. Hayes, A. F. J. Levi, and W. Wiegmann, "Hot-electron spectroscopy of GaAs," Physical Review Letters, vol. 54, no. 14, article 1570, 1985.

[56] A. F. J. Levi, J. R. Hayes, P. M. Platzman, and W. Wiegmann, "Injected-hot-electron transport in GaAs," Physical Review Letters, vol. 55, no. 19, pp. 2071-2073, 1985.

[57] A. Braunstein, M. Braunstein, G. S. Picus, and C. A. Mead, "Photoemissive determination of barrier shape in tunnel junctions," Physical Review Letters, vol. 14, no. 7, pp. 219-221, 1965.

[58] A. Prem and V. V. Kresin, "Photoionization profiles of metal clusters and the Fowler formula," Physical Review A, vol. 85, no. 2, Article ID 025201, 4 pages, 2012.

[59] F. Williams and A. J. Nozik, "Solid-state perspectives of the photoelectrochemistry of semiconductor-electrolyte junctions," Nature, vol. 312, no. 5989, pp. 21-27, 1984.

[60] M. Cinchetti, K. Heimer, J.-P. Wüstenberg et al., "Determination of spin injection and transport in a ferromagnet/organic semiconductor heterojunction by two-photon photoemission," Nature Materials, vol. 8, no. 2, pp. 115-119, 2009.

[61] W. Wang, G. Lüpke, M. Di Ventra et al., "Coupled electron-hole dynamics at the $\mathrm{Si} / \mathrm{SiO}_{2}$ interface," Physical Review Letters, vol. 81, no. 19, pp. 4224-4227, 1998. 


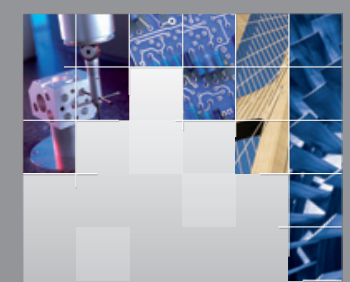

\section{Enfincering}




The Scientific World Journal

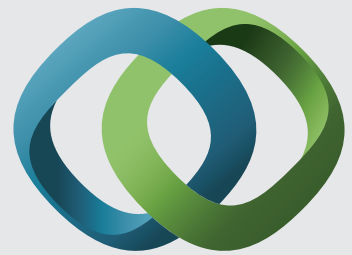

\section{Hindawi}

Submit your manuscripts at

http://www.hindawi.com
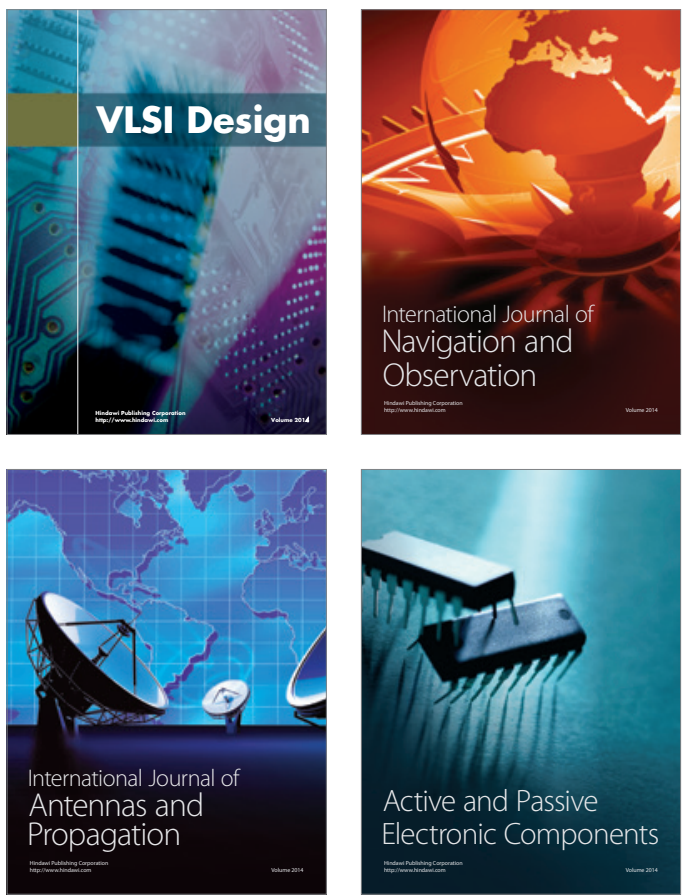
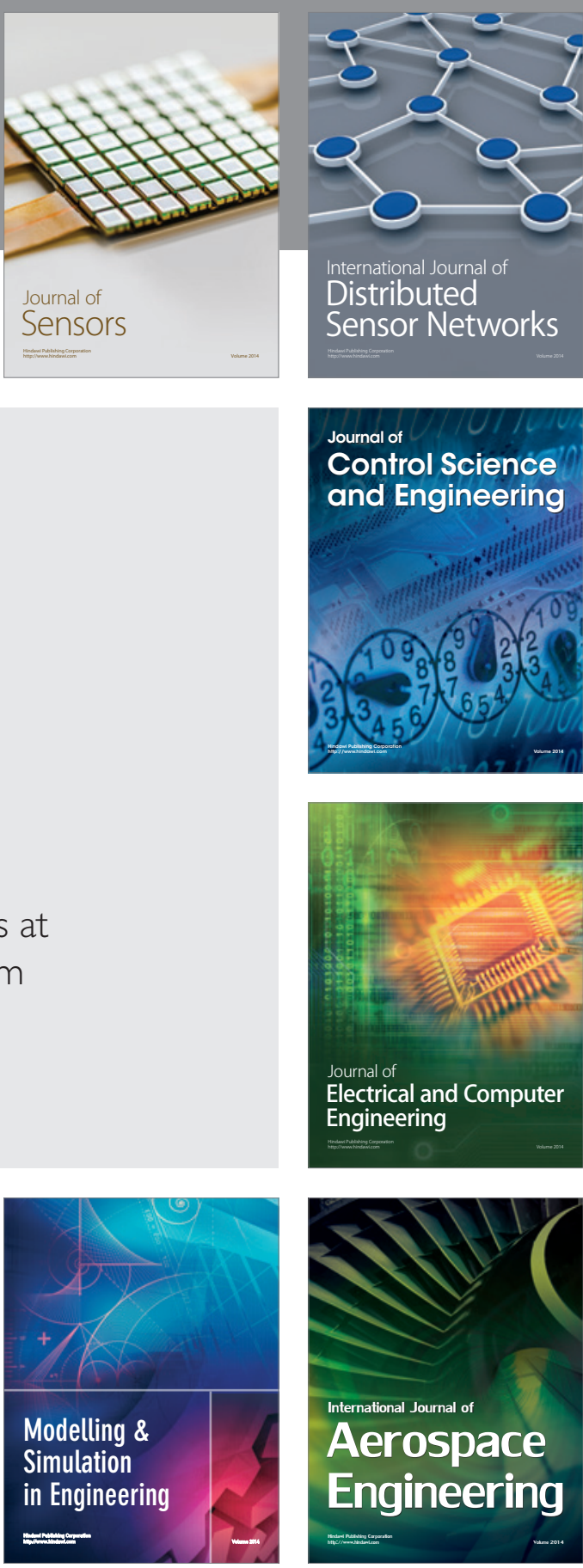

International Journal of

Distributed

Sensor Networks

Journal of

Control Science

and Engineering
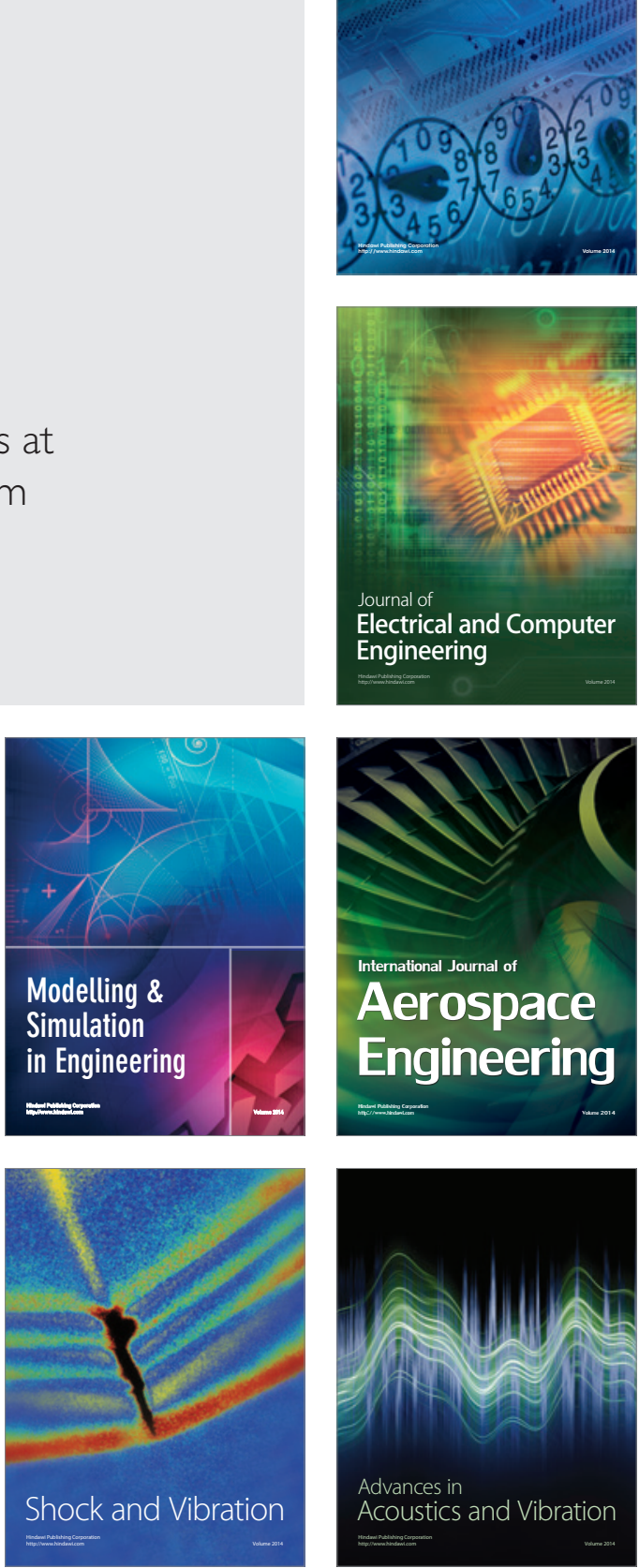